\title{
Adaptive Control Design for a Boost Inverter ${ }^{\underline{2}}$
}

\author{
Carolina Albea*,1 \\ Ingeniería de Sistemas y Automática. Universidad de Sevilla, Spain \\ Francisco Gordillo, ${ }^{2}$ \\ Ingeniería de Sistemas y Automática. Universidad de Sevilla, Spain \\ Carlos Canudas-de-Wit, ${ }^{3}$ \\ GIPSA-Lab, CNRS, France, Spain
}

\begin{abstract}
In this paper, a novel control strategy for a nonlinear boost inverter is proposed. The idea is based on generating an autonomous oscillator that does not need an external reference signal. This aim is achieved by using energyshaping methodology with a suitable Hamiltonian function which defines the desired system behavior. A phase controller is added to the control law in order to achieve $180^{\circ}$-synchronization between both parts of the circuit as well as synchronize the voltage output with a pre-specified signal, e.g. synchro-

\footnotetext{
Whis research was funded by the MICINN-FEDER grant DPI2009-09961.

Email addresses: calbea@cartuja.us.es (Carolina Albea), gordillo@esi.us.es (Francisco Gordillo,), carlos.canudas-de-wit@gipsa-lab.inpg.fr (Carlos Canudas-de-Wit, )

${ }^{1}$ Escuela Superior de Ingenieros, Avd de los Descubrimientos s/n, 41092, Sevilla, Spain, Tlf: +34954 4874 87, Fax:+34 954487340

${ }^{2}$ Escuela Superior de Ingenieros, Avd de los Descubrimientos s/n, 41092, Sevilla, Spain, Tlf: +34 954487345 , Fax:+34 954487340

${ }^{3}$ GIPSA-Lab, BP. 46, 38402, ST Martin d'Hères, France, Tlf: +33 476.82.63.80, Fax: +33476.82 .63 .88$
} 
nization with the electrical grid. An adaptive control is designed for dealing with the common problem of unknown load. In order to analyze the stability of the full system, singular perturbation approach is used. The resulting control is tested by means of simulations.

Key words: Adaptive control, Inverters, Nonlinear circuits, Limit cycles, Synchronization, Singular perturbation method.

\section{Introduction}

The control of switched-mode power converters (SMPC) with AC output is usually accomplished by tracking a reference (sinusoidal) signal (see Biel et al., 2004; de Souza et al., 2000; Sanchís et al., 2005; Vázquez et al., 2003). The use of this external signal makes the closed-loop control system nonautonomous and thus, causes its analysis to be more involved than if it were autonomous.

In these systems, the control objective can be seen as the generation of a stable limit cycle with a given amplitude and frequency. If a control law is able to produce such a limit cycle, alternating current will be generated without the need to introduce a time-dependent reference signal. The generation of limit cycles for producing self-oscillations has been successfully applied to electro-mechanical systems (Gómez-Estern et al., 2002; Gordillo et al., 2002). Some applications to electronic converters are (Aracil and Gordillo, 2002; Gordillo et al., 2004), where a three-phase UPS and a boost converter are controlled using this method.

The idea behind (Biel et al., 2001) is similar but there a sliding mode controller is proposed, which yields the typical drawback of this kind of con- 
troller: the switching frequency is variable.

In this paper, this approach is applied to a novel boost inverter (Albea et al., 2006). This novel boost inverter is especially interesting because it can generate alternating current since it produces an oscillating voltage centered around zero and achieves negative voltages.

In this paper, it is first shown that the direct application of the approach proposed in Aracil and Gordillo (2002) and Gordillo et al. (2004) does not fulfil the objective due to the lack of anti-synchronization ${ }^{4}$ between both parts of the circuit. In order to achieve anti-synchronization, a phase controller in an external loop is added to the previous control law. This approach is also applied to synchronize ${ }^{5}$ the output with a given signal. An example of such a configuration is the synchronization of the boost inverter with the electrical grid in order to achieve a satisfactory power factor.

Some simulations of industrial applications are presented in this paper in order to validate the circuit performance.

Once the system is controlled, it is well known in industrial applications that, the load can be unknown or suffer perturbations. This problem in SMPC is usually dealt with by using adaptation mechanisms along with other techniques such as feedback stabilization (H. et al., 1993) input-output feedback linearization (Hadri-Hamida et al., 2008), backstepping (Ríos-Bolívar et al., 1996; Sira-Ramírez et al., 1997), a grid-point modeling approach (Ng

\footnotetext{
${ }^{4}$ In this paper it is said that two sinusoidal signals of the same frequency are antisynchronized, or in anti-phase, when the phase shift between them is equal to $180^{\circ}$.

${ }^{5}$ In this paper it is said that two sinusoidal signals of the same frequency are synchronized when the phase shift between them is equal to $0^{\circ}$.
} 
et al., 1996), a sliding mode (Carrasco et al., 1997; Sira-Ramírez and RíosBolívar, 1994; Tan et al., 2005), predictive control (MeVay and Sarpeshkar, 2003), or fuzzy logic (Elmas et al., 2007).

In Pagano et al. (2005), an adaptive control is obtained for the case of the boost converter controlled using a law based on the oscillation generation approach. It is computed using passivity arguments. This approach is not easily applicable to the boost inverter because its model is more involved than that of the converter of Pagano et al. (2005).

The main goal of this study is to find a load-adaptation mechanism in the case of the boost inverter. The idea is to design a state observer for some of the converter variables even when the state variables are measured, in order to estimate the load. This provides a fast, successful adaptation of the load parameter (Albea et al., 2007). This approach is applied by simulation to a real industrial case.

In order to analyze the stability of the full system singular perturbation analysis, Khalil (2002) and Kokotovic et al. (1999) are used. For the sake of simplicity, the phase controller is not considered in this analysis. The resulting adaptive control is tested by means of simulations.

The rest of the work is organized as follows: in Section 2 the model of the double boost converter (boost inverter) is presented. Section 3 shows the general idea of generating oscillating behavior by means of the generation of a limit cycle through energy shaping. This idea yields a controller for the boost inverter, but it is shown that the behavior is not acceptable due to a lack of synchronization. In Section 4 a phase controller is added to achieve the synchronization of an isolated boost inverter as well as the synchronization of 
the boost inverter with a pre-specified signal. Section 5 deals with the main contribution of this paper: the unknown-load case, which is solved by means of adaptation mechanism design. Section 6 is devoted to the stability analysis and presents some simulation results. Finally, conclusions are outlined in Section 7 .

\section{Boost Inverter Model}

The boost inverter (Cáceres and Barbi, 1999) can generate an AC output voltage larger than its DC input, i. e., it is an voltage elevator. It is made up of two DC-DC converters ${ }^{6}$ and a load connected differentially across them (see Fig. 1). Each converter produces a DC-biased sine wave output, $v_{1}$ and $v_{2}$, so that each source generates a unipolar voltage. Voltages $v_{1}$ and $v_{2}$ should present a phase shift equal to $180^{\circ}$, which maximizes the voltage excursion across the load. In this way it is possible to generate an oscillatory signal without bias. The circuit implementation is shown in Fig. 2.

In order to simplify the analysis, a part of the boost inverter is replaced by a voltage source as shown in Fig. 3, and these results are extrapolated to the full inverter. Note, that this replacement shows more clearly the bidirectional current of each boost DC-DC converter.

\subsection{System Description}

Let us assume that:

\footnotetext{
${ }^{6}$ Throughout this paper, each part of the boost inverter will be referred as "boost DC-DC converter" since each part is a normal boost converter that is commonly used as a DC-DC converter. Nevertheless, it should be taken into account that, in the boost inverter, each part does not act as a DC-DC converter.
} 


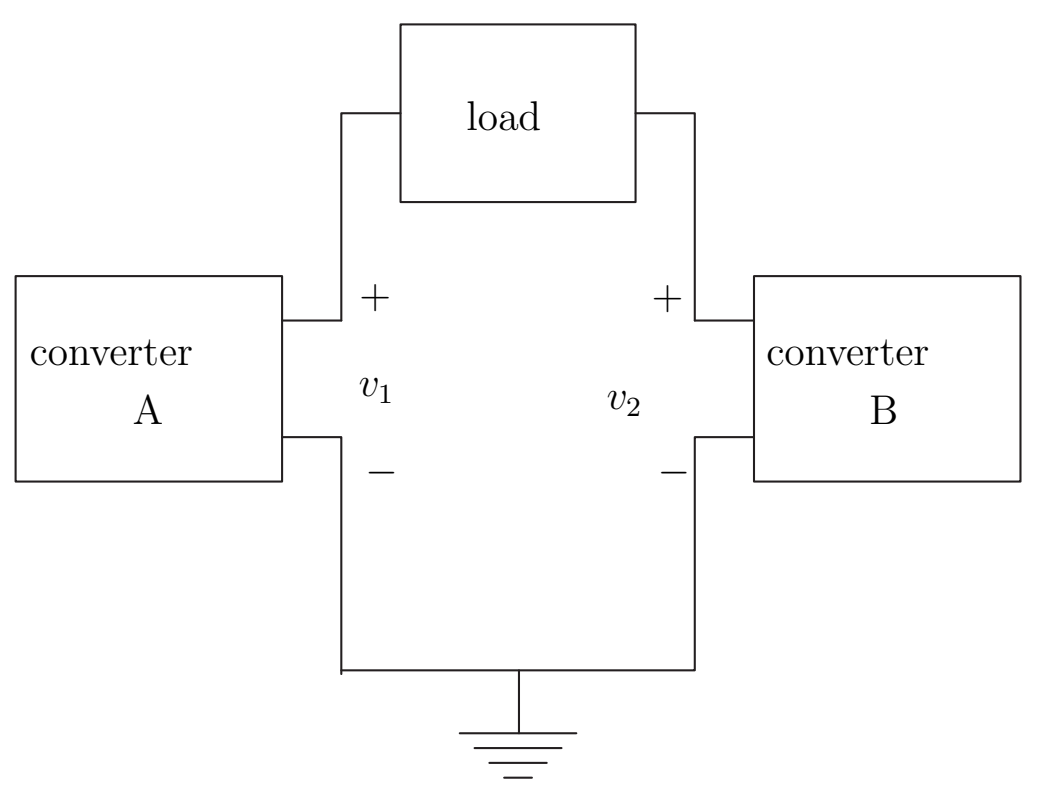

Figure 1: Basic representation of the boost inverter.

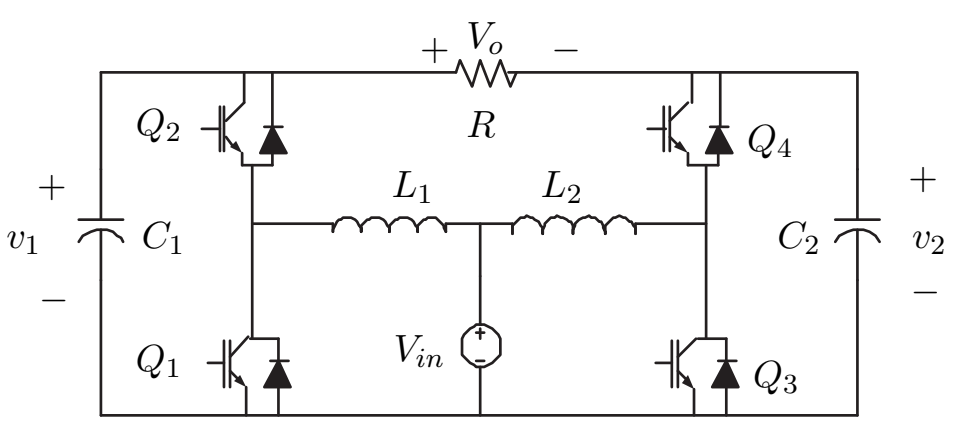

Figure 2: Boost inverter model. 


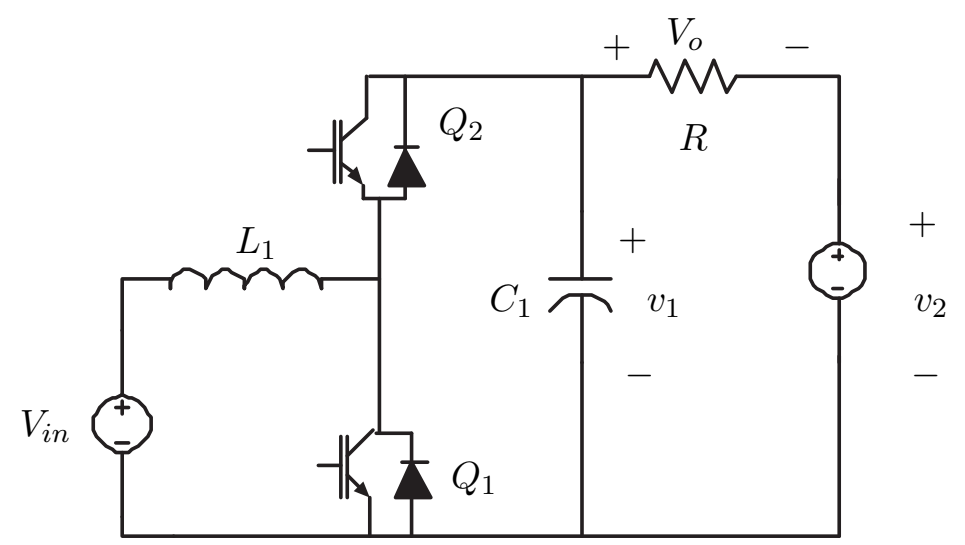

Figure 3: Boost inverter model with replacement of a voltage source.

- all the components are ideal and the currents of the converter are continuous,

- the power supply is free of sinusoidal ripple and is known,

- the converter operates at a high-switching frequency characteristic of SMPC,

- the inductances $L_{1}=L_{2}$, and the capacitances $C_{1}=C_{2}$, are known and symmetric,

- $v_{1}$ and $v_{2}$ are positive, sinusoidal voltages.

The circuit in Fig. 3 is driven by the transistor ON/OFF inputs $Q_{i}$. This yields two modes of operation illustrated in Fig. 4. Formally, this yields a switched model. For control purposes, it is common to use an average model described in terms of the mean currents and voltages values. This model is more suitable for control because it is described by an averaged, 
smooth, nonlinear, continuous-time ODE. This averaging process yields the normalized model described below.
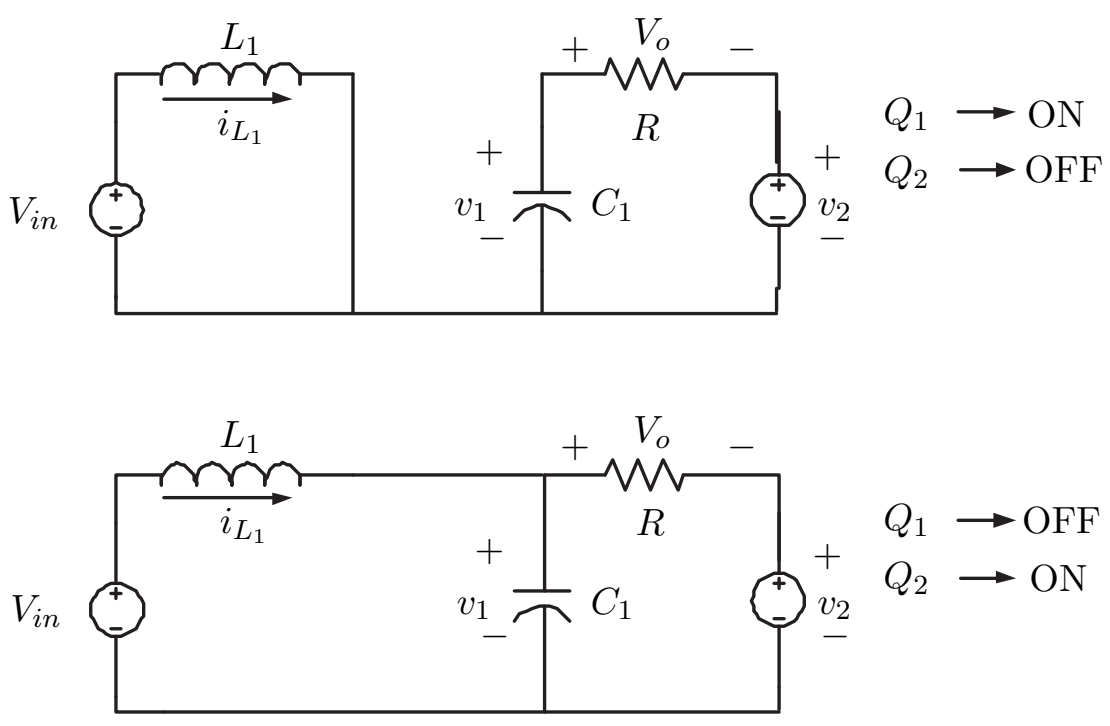

Figure 4: Operation modes.

If the control variable, $q$, is defined as $q=0$ when $Q_{1}=\mathrm{OFF}$ and $Q_{2}=\mathrm{ON}$, and $q=1$, when $Q_{1}=\mathrm{ON}$ and $Q_{2}=\mathrm{OFF}$, the converter dynamic equations are

$$
\begin{aligned}
L_{1} \frac{d i_{L_{1}}}{d t} & =-v_{1}+q v_{1}+V_{i n} \\
C_{1} \frac{d v_{1}}{d t} & =i_{L_{1}}-q i_{L_{1}}-\frac{v_{1}}{R}+\frac{v_{2}}{R} .
\end{aligned}
$$

Let us take $\underline{u}=1-q$ as the control action in equations (1)-(2); getting

$$
\begin{aligned}
& L_{1} \frac{d i_{L_{1}}}{d t}=-\underline{u} v_{1}+V_{i n} \\
& C_{1} \frac{d v_{1}}{d t}=\underline{u} i_{L_{1}}-\frac{v_{1}}{R}+\frac{v_{2}}{R},
\end{aligned}
$$

where $\underline{u}$ is the control variable, which can only take two values $\underline{u} \in\{0,1\}$. Nevertheless, as is usual (Middlebrook and Ćuk, 1977), let us consider its av- 
erage value by $u(t)=\frac{1}{T} \int_{t-T}^{t} \underline{u}(s) d s$ where $T$ is the switching period. Therefore, $u$ is a continuous variable $u \in[0,1]$.

\subsection{Normalized Average Model}

In order to simplify the study, and assuming a resistive load, system $(3)-(4)$ is normalized in terms of the averaged current $x_{1}$ and the averaged voltages $x_{2}$ and $x_{4}$ by using the following change of variables

$$
\begin{aligned}
x_{1} & =\frac{1}{V_{i n}} \sqrt{\frac{L_{1}}{C_{1}}} i_{L_{1}} \\
x_{2} & =\frac{v_{1}}{V_{i n}} \\
x_{4} & =\frac{v_{2}}{V_{i n}}
\end{aligned}
$$

and defining

$$
\tilde{t}=\omega_{0} t
$$

as a new time variable with

$$
\omega_{0}=\frac{1}{\sqrt{L_{1} C_{1}}}
$$

which yields

$$
\begin{aligned}
& \dot{x}_{1}=-u x_{2}+1 \\
& \dot{x}_{2}=u x_{1}-a x_{2}+a x_{4},
\end{aligned}
$$

where $a=\frac{1}{R} \sqrt{\frac{L_{1}}{C_{1}}}$. Note that $\dot{x}_{1}$ and $\dot{x}_{2}$ are derivatives of $x_{1}$ and $x_{2}$ with respect to $\tilde{t}$.

If $u$ is eliminated in (10)-(11), it is got

$$
x_{1}\left(1-\dot{x}_{1}\right)=x_{2}\left(\dot{x}_{2}+a x_{2}-a x_{4}\right) .
$$


This equation is an implicit equation, which relates the state variables $\left(x_{2}\right.$, $x_{4}$ ) and their derivatives and does not depend on the control signal $u$. Note that $x_{4}$ can be considered an exogenous input in system (10)-(11). Equation (12) can be understood as the internal dynamics of the system. If $\dot{x}_{1}=0$ and $\dot{x}_{2}=0$, the equilibrium manifold is $x_{1}=a x_{2}\left(x_{2}-x_{4}\right)$. In this way, the internal dynamics of system (10)-(11) given by (12) acts as a constraint on the state of the system.

From Eq. (12), it is possible to see that given $x_{4}$ and only controlling $x_{1}$, variable $x_{2}$ can be indirectly controlled ${ }^{7}$. Moreover, the stability of the system is maintained (Fossas and Olm-Miras, 2002).

\section{Energy Shaping Control}

\subsection{Generation of Oscillations by Energy Shaping}

The generation of alternating current in power electronic converters can be achieved by generating a stable limit cycle without the need to introduce a reference signal. To do this, an oscillatory target system may be defined and by matching its equations and system equations (10)-(11) a control law can be obtained. In order to define the target system, consider the following energy-like function

$$
H_{0}\left(\eta_{1}, \eta_{2}\right)=\frac{1}{4} \Gamma^{2}\left(\eta_{1}, \eta_{2}\right)
$$

where $\eta_{1}$ and $\eta_{2}$ are state variables and $\Gamma\left(\eta_{1}, \eta_{2}\right)=\omega^{2}\left(\eta_{1}-\eta_{10}\right)^{2}+\left(\eta_{2}-\right.$ $\left.\eta_{20}\right)^{2}-\mu$. Parameters $\omega, \eta_{10}, \eta_{20}$ and $\mu>0$ should be chosen so that the

\footnotetext{
${ }^{7}$ For the full system, it is had: $x_{1}\left(1-\dot{x}_{1}\right)=x_{2}\left(\dot{x}_{2}+a\left(x_{2}-x_{4}\right)\right)$ and $x_{3}\left(1-\dot{x}_{3}\right)=$ $x_{4}\left(\dot{x}_{4}+a\left(x_{4}-x_{2}\right)\right)$. Thus, controlling $x_{1}$ and $x_{3}$, the desired behaviors for $x_{2}$ and $x_{4}$ can be obtained.
} 
closed curve $\Gamma=0$ defines the desired behavior. This curve is an ellipse centered at point $\left(\eta_{10}, \eta_{20}\right)$. A dynamical system can be defined such that this closed curve is its limit set. This can be reached by adopting $H_{0}$ as a Hamiltonian function (Aracil and Gordillo, 2002; Pagano et al., 2005), and defining the Hamiltonian dynamical system

$$
\left[\begin{array}{c}
\dot{\eta}_{1} \\
\dot{\eta}_{2}
\end{array}\right]=\left[\begin{array}{cc}
-k_{a_{1}} & \frac{1}{\Gamma} \\
-\frac{1}{\Gamma} & -k_{a_{2}}
\end{array}\right]\left[\begin{array}{l}
\frac{\partial H_{0}}{\partial \eta_{1}} \\
\frac{\partial H_{0}}{\partial \eta_{2}}
\end{array}\right],
$$

which, after using (13), results in

$$
\begin{aligned}
& \dot{\eta}_{1}=\left(\eta_{2}-\eta_{20}\right)-k_{a_{1}} \omega^{2}\left(\eta_{1}-\eta_{10}\right) \Gamma \\
& \dot{\eta}_{2}=-\omega^{2}\left(\eta_{1}-\eta_{10}\right)-k_{a_{2}}\left(\eta_{2}-\eta_{20}\right) \Gamma .
\end{aligned}
$$

Taking into account that

$$
\dot{H}_{0}=-\Gamma^{2}\left(k_{a_{1}} \omega^{4}\left(\eta_{1}-\eta_{10}\right)^{2}+k_{a_{2}}\left(\eta_{2}-\eta_{20}\right)^{2}\right) \leq 0,
$$

by using the LaSalle invariance principle it can be seen that, for all initial conditions except the center of the ellipse, the trajectories of the system tend to the curve $\Gamma=0$.

The behavior of this target system corresponds to the desired sinusoidal behavior for the DC-AC converter. Constants $\omega, \eta_{10}, \eta_{20}$ and $\mu$ are design parameters for the frequency bias and amplitude of the desired behavior while $k_{a_{1}}$ and $k_{a_{2}}$ define the speed of the transient response.

Note that $\dot{\eta}_{1}$ and $\dot{\eta}_{2}$ are in this case derivatives of $\eta_{1}$ and $\eta_{2}$ with respect to $\tilde{t}$, in order to work with the normalized averaged model (10)-(11).

\subsection{Controller Design}

System (10)-(11) can not be directly transformed to the form of system (15)-(16), but this can be done using the new change of coordinates given 
below:

$$
\begin{aligned}
& \zeta_{1}=\frac{x_{1}^{2}+x_{2}^{2}}{2} \\
& \zeta_{2}=x_{1}-a x_{2}^{2}+a x_{2} x_{4}+\zeta_{20}
\end{aligned}
$$

where $\zeta_{20}$ is an offset term that will be a tuning parameter. From (18)-(19), it is easy to see that

$$
\begin{aligned}
\dot{\zeta}_{1}= & \zeta_{2}-\zeta_{20} \\
\dot{\zeta}_{2}= & 1+2 a^{2} x_{2}^{2}-3 a^{2} x_{4} x_{2}+a^{2} x_{4}^{2}+a x_{2} \dot{x_{4}} \\
& -u\left(x_{2}+2 a x_{1} x_{2}-a x_{4} x_{1}\right) .
\end{aligned}
$$

It is not easy to obtain simple relationships $x_{1}=f\left(\zeta_{1}, \zeta_{2}\right)$ and $x_{2}=$ $f\left(\zeta_{3}, \zeta_{4}\right)$ from (18)-(19) due to the quadratic terms. Nevertheless, this change of variables is a diffeomorphism if and only if $x_{2}+2 a x_{1} x_{2}-a x_{4} x_{1} \neq 0$, as follows from the inverse function theorem. This constraint restricts the domain of attraction of the desired limit cycle when the controller obtained below is applied. Such a domain of attraction is analyzed in (Albea and Gordillo, 2007).

Looking at target system structure (15)-(16) and comparing it with (20)(21) the choice $k_{a_{1}}=0$ is obvious, resulting in the target system

$$
\begin{aligned}
& \dot{\zeta}_{1}=\zeta_{2}-\zeta_{20} \\
& \dot{\zeta}_{2}=-\omega^{2}\left(\zeta_{1}-\zeta_{10}\right)-k \Gamma\left(\zeta_{2}-\zeta_{20}\right),
\end{aligned}
$$

where, for sake of simplicity, $k_{a_{2}}$ has been denoted as $k$. The attraction of curve $\Gamma=0$ can still be proved by the LaSalle invariance principle. 
The control law, $u$, that matches (20)-(21) and (22)-(23) is

$$
\begin{aligned}
u= & \frac{1+2 a^{2} x_{2}^{2}-3 a^{2} x_{4} x_{2}+a^{2} x_{4}^{2}+a x_{2} \dot{x_{4}}}{x_{2}+2 a x_{1} x_{2}-a x_{4} x_{1}} \\
& +\frac{k \Gamma\left(\zeta_{2}-\zeta_{20}\right)+\omega^{2}\left(\zeta_{1}-\zeta_{10}\right)}{x_{2}+2 a x_{1} x_{2}-a x_{4} x_{1}} .
\end{aligned}
$$

Indeed, $u$ varies dependently on $x$, as is noted from Eq. (18)-(19). This controller has several problems. First, the denominator in (24) may be zero (this is the same necessary condition for (20)-(21) to be a diffeomorphism). Furthermore, in other cases, the resulting $u$ can violate the constraint $0 \leq$ $u \leq 1$. In Albea and Gordillo (2007) an estimation for the region of attraction of the desired limit cycle is obtained by taking these problems into account. It is assumed that a starting strategy will bring the state of the system into this region of attraction (Alonso et al., 2000; Malinowski et al., 2003; Zhu et al., 2000).

Parameters $\eta_{10}, \eta_{20}$ and $\mu$ have to be defined as a function of the desired behavior. To do so, it is necessary to obtain an analytical expression of the desired objective curve in plane $x_{1}-x_{2}$. Assume that the desired time evolutions for $x_{2}$ and $x_{4}$ are

$$
\begin{aligned}
& x_{2}^{*}=A \sin \omega t+B \\
& x_{4}^{*}=-A \sin \omega t+B,
\end{aligned}
$$

where $A, B$ and $\omega$ take pre-specified values to obtain the desired evolution for $v_{1}$ and $i_{L_{1}}$ using (5)-(7), (8) and (9). In addition, note that these desired evolutions allow us to remove the bias in the output. The origin of time in (25)-(26) is arbitrary so no phase shift value is imposed (signal synchronization will be achieved below). Assume that the desired steady state for $x_{1}$ can 
be approximated by

$$
x_{1}^{*}=a \alpha_{0}+\alpha_{1} \cos \omega t+\beta_{1} \sin \omega t
$$

This assumption is very common in the field of electronics field (see Fossas and Olm-Miras, 2002; Dixon and Ooi, 1988; Griño et al., 2002; Biel et al., 2004).

By substituting (25)-(26) and (27) in (12)

$$
\begin{array}{r}
a \alpha_{0}+\left(\beta_{1}+a \alpha_{0} \alpha_{1} \omega\right) \sin \omega t+\left(\alpha_{1}-a \alpha_{0} \beta_{1} \omega\right) \cos \omega t \\
+\frac{1}{2} \omega\left(\alpha_{1}^{2}-\beta_{1}^{2}\right) \sin 2 \omega t-\alpha_{1} \beta_{1} \omega \cos 2 \omega t \\
=a A^{2}-2 a A B \sin \omega t-\omega A B \cos \omega t+ \\
\frac{1}{2} \omega A^{2} \sin 2 \omega t-a A^{2} \cos 2 \omega t
\end{array}
$$

If the second order harmonics are neglected, the corresponding coefficients can be equated:

$$
\begin{aligned}
a \alpha_{0} & =a A^{2} \\
\beta_{1}+a \alpha_{0} \alpha_{1} \omega & =-2 a A B \\
\alpha_{1}-a \alpha_{0} \beta_{1} \omega & =-\omega A B .
\end{aligned}
$$

When this system is resolved for $\alpha_{0}, \alpha_{1}$ and $\beta_{1}$,

$$
\begin{aligned}
& \alpha_{0}=A^{2} \\
& \alpha_{1}=\frac{\omega A B\left(2 a^{2} A^{2}+1\right)}{1+a^{2} A^{4} \omega^{2}} \\
& \beta_{1}=-\frac{a A B\left(\omega^{2} A^{2}-2\right)}{1+a^{2} A^{4} \omega^{2}} .
\end{aligned}
$$

The next question is to show that the desired behavior for $\zeta_{1}$ and $\zeta_{2}$ is an ellipse and to define the ellipse parameters $\left(\omega, \zeta_{10}, \zeta_{20}\right.$ and $\left.\mu\right)$ in terms 
of the desired behavior for $x_{2}$. In order to do this, it is necessary to obtain the desired evolution for $\zeta_{2}$ by applying the change of variables (18)-(19) to (25)-(26) and (27).

$$
\begin{aligned}
\zeta_{1}= & \frac{1}{2}\left[\left(a \alpha_{0}+\alpha_{1} \cos \omega t+\beta_{1} \sin \omega t\right)^{2}+\right. \\
& \left.(A \sin \omega t+B)^{2}\right] \\
\zeta_{2}= & a \alpha_{0}+\alpha_{1} \cos \omega t+\beta_{1} \sin \omega t-a(A \sin \omega t+B)^{2}+ \\
& a\left(-A^{2} \sin ^{2} \omega t+B^{2}\right)+\zeta_{20}
\end{aligned}
$$

Expanding these expressions in Fourier terms yields

$$
\begin{aligned}
\zeta_{1}= & \zeta_{1}^{(0)}+\zeta_{1}^{(11)} \cos \omega t+\zeta_{1}^{(12)} \sin \omega t+\zeta_{1}^{(21)} \cos 2 \omega t \\
& +\zeta_{1}^{(22)} \sin 2 \omega t \\
\zeta_{2}= & \zeta_{2}^{(0)}+\zeta_{2}^{(11)} \cos \omega t+\zeta_{2}^{(12)} \sin \omega t+\zeta_{2}^{(21)} \cos 2 \omega t \\
& +\zeta_{2}^{(22)} \sin 2 \omega t
\end{aligned}
$$

By equating (31)-(32) with (33)-(34) the following Fourier coefficients 
are obtained

$$
\begin{aligned}
\zeta_{1}^{(0)} & =\frac{2 a^{2} \alpha_{0}^{2}+\alpha_{1}^{2}+\beta_{1}^{2}+A^{2}+2 B^{2}}{4} \\
\zeta_{1}^{(11)} & =a \alpha_{0} \alpha_{1} \\
\zeta_{1}^{(12)} & =a \alpha_{0} \beta_{1}+A B \\
\zeta_{1}^{(21)} & =\frac{\alpha_{1}^{2}-\beta_{1}^{2}-A^{2}}{4} \\
\zeta_{1}^{(22)} & =\frac{\alpha_{1} \beta_{1}}{2} \\
\zeta_{2}^{(0)} & =\zeta_{20} \\
\zeta_{2}^{(11)} & =\alpha_{1} \\
\zeta_{2}^{(12)} & =\beta_{1}-2 a A B \\
\zeta_{2}^{(21)} & =a A^{2} \\
\zeta_{2}^{(22)} & =0 .
\end{aligned}
$$

Assuming that the double frequency terms $\zeta_{1}^{(21)}, \zeta_{1}^{(22)}, \zeta_{2}^{(21)}$ and $\zeta_{2}^{(22)}$ can be neglected, these expressions can be approximated by an ellipse in the plane $\zeta_{1}, \zeta_{2}$ since (31)-(32) yields

$$
\begin{aligned}
& \omega \zeta_{1}^{(11)}=-\zeta_{2}^{(12)} \\
& \omega \zeta_{1}^{(12)}=\zeta_{2}^{(11)} .
\end{aligned}
$$

The parameters of this ellipse are given by

$$
\begin{aligned}
\zeta_{10} & =\zeta_{1}^{(0)} \\
\zeta_{20} & =\zeta_{2}^{(0)} \\
\mu & =\omega^{2}\left(\left(\zeta_{1}^{(11)}\right)^{2}+\left(\zeta_{1}^{(12)}\right)^{2}\right) .
\end{aligned}
$$




\subsection{Control Law for the Full Converter}

In this section the previous control law is modified in order to take into account the fact that the boost inverter is composed of two DC-DC converters and, thus, it has two control signals. The model of the system is

$$
\begin{aligned}
L_{1} \frac{d i_{L_{1}}}{d t} & =-u_{1} v_{1}+V_{i n} \\
C_{1} \frac{d v_{1}}{d t} & =u_{1} i_{L_{1}}-\frac{v_{1}}{R}+\frac{v_{2}}{R} \\
L_{2} \frac{d i_{L_{2}}}{d t} & =-u_{2} v_{2}+V_{i n} \\
C_{2} \frac{d v_{2}}{d t} & =u_{2} i_{L_{2}}+\frac{v_{1}}{R}-\frac{v_{2}}{R} .
\end{aligned}
$$

By comparing systems (40)-(43) and (3)-(4) there is a similar structure for the pairs of current and voltage of both boost DC-DC converters. Therefore, the two control laws are easily obtained. Control law $u_{2}$ is obtained by using symmetry. The control laws are

$$
\begin{aligned}
u_{1}= & \frac{1+2 a^{2} x_{2}^{2}-3 a^{2} x_{2} x_{4}+a^{2} x_{4}^{2}+a^{2} x_{2} \dot{x}_{4}}{x_{2}+2 a x_{1} x_{2}-a x_{4} x_{1}} \\
& +\frac{k_{1} \Gamma_{1}\left(\zeta_{2}-\zeta_{20}\right)+\omega^{2}\left(\zeta_{1}-\zeta_{10}\right)}{x_{2}+2 a x_{1} x_{2}-a x_{4} x_{1}} \triangleq \kappa_{1}(x) \\
u_{2}= & \frac{1+2 a^{2} x_{4}^{2}-3 a^{2} x_{2} x_{4}+a^{2} x_{2}^{2}+a^{2} x_{4} \dot{x}_{2}}{x_{4}+2 a x_{3} x_{4}-a x_{2} x_{3}} \\
& +\frac{k_{2} \Gamma_{2}\left(\zeta_{4}-\zeta_{40}\right)+\omega^{2}\left(\zeta_{3}-\zeta_{30}\right)}{x_{4}+2 a x_{3} x_{4}-a x_{2} x_{3}} \triangleq \kappa_{2}(x),
\end{aligned}
$$

where

$$
\begin{aligned}
& \Gamma_{1}\left(\zeta_{1}, \zeta_{2}\right)=\omega^{2}\left(\zeta_{1}-\zeta_{10}\right)^{2}+\left(\zeta_{2}-\zeta_{20}\right)^{2}-\mu \\
& \Gamma_{2}\left(\zeta_{3}, \zeta_{4}\right)=\omega^{2}\left(\zeta_{3}-\zeta_{30}\right)^{2}+\left(\zeta_{4}-\zeta_{40}\right)^{2}-\mu .
\end{aligned}
$$

The expressions for derivatives $\dot{x}_{2}$ and $\dot{x}_{4}$ are taken directly from the normalized equations of the boost inverter. 


\subsection{Simulation Results}

The following simulations shows an industrial case. It is desired to obtain an output voltage $V_{o}=220 \frac{2}{\sqrt{2}} \sin (100 \pi t)$ from an input voltage $V_{i n}=48 \mathrm{~V}$.

These simulations are obtained considering, $R=50 \Omega, L=600 \mu H, C=$ $600 \mu \mathrm{F}$. The desired frequency and voltage amplitude are $50 \mathrm{~Hz}$ and $220 \mathrm{~V}_{\text {rms }}$, respectively.

Parameter $A$ in Eqs. (25)-(26) has to be the half of the desired output voltage amplitude and $B$ is chosen so that $x_{2}$ and $x_{4}$ are always positive. In order to obtain this voltage, the parameters are $A=3.33$ and $B=5.42$ with $\omega=0.188$ in the normalized variables $\left(x_{1}, x_{2}\right)$.

Figure 5 shows the results of a simulation using a commutation frequency of $50 \mathrm{KHz}$ and employing a sample time of $0.1 T \mathrm{~s}$, where $T$ is the commutation frequency period. Both DC-DC converters achieve the desired limit cycle.

Figure 6 shows the boost inverter output voltage. In this figure only the steady state behavior is shown. It can be seen that the desired amplitude is not achieved. The reason is that the previous design does not force the phase shift between signals $v_{1}$ and $v_{2}$ to be in anti-phase. Figure 7 shows that, as a result, this goal is not achieved. Thus, the system is extremely sensitive to the initial values of the desired signals: $x_{2}$ and $x_{4}$. The next section deals with this problem.

\section{Synchronization Problem}

The controllers developed above for boost inverters do not synchronize the two parts of the circuit with a phase shift of $180^{\circ}$ since each one independently 
a)

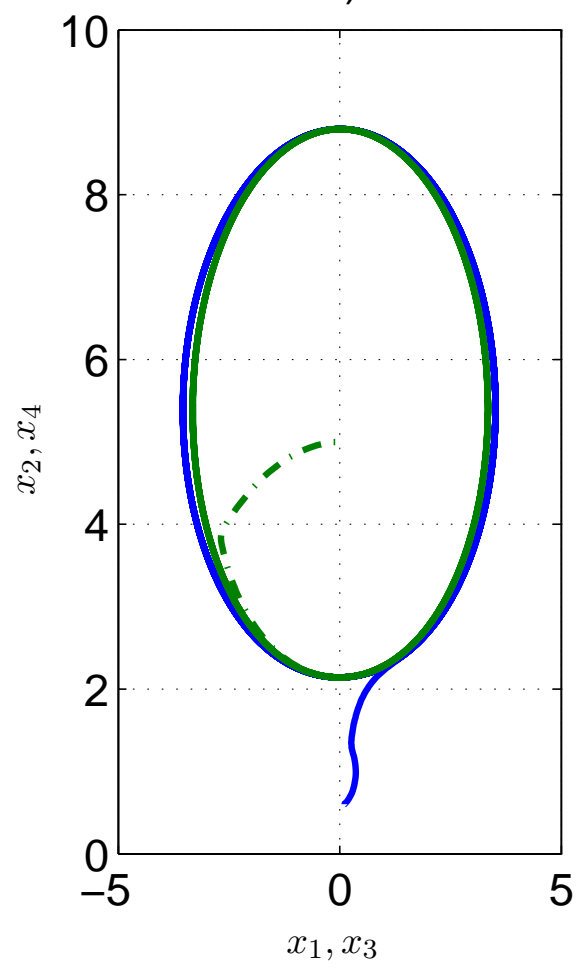

b)

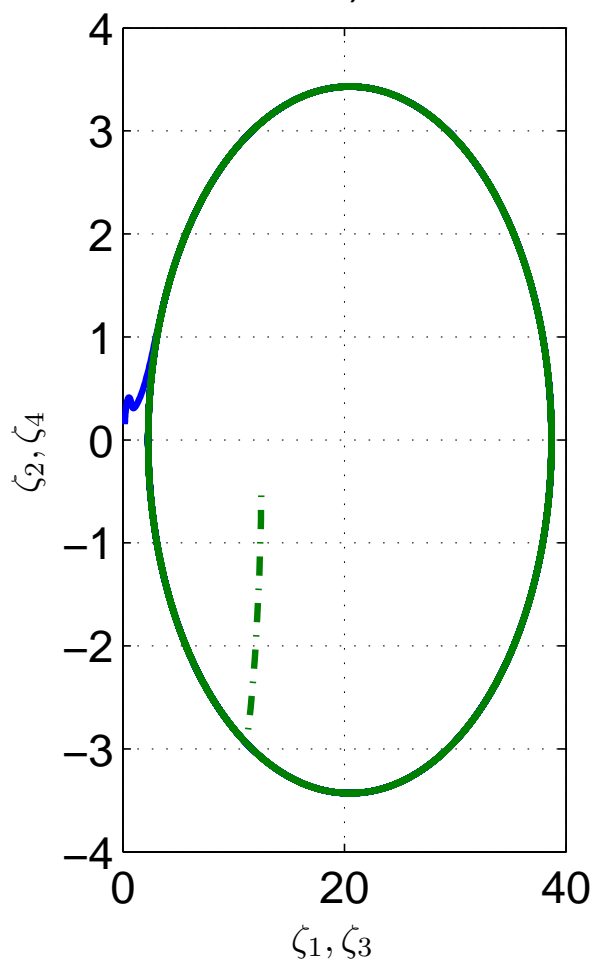

Figure 5: a) Evolution of $\left(x_{1}, x_{2}\right)$ (solid) and $\left(x_{3}, x_{4}\right)$ (dashed); b) evolution of $\left(\zeta_{1}, \zeta_{2}\right)$ (solid) and $\left(\zeta_{3}, \zeta_{4}\right)$ (dashed). 


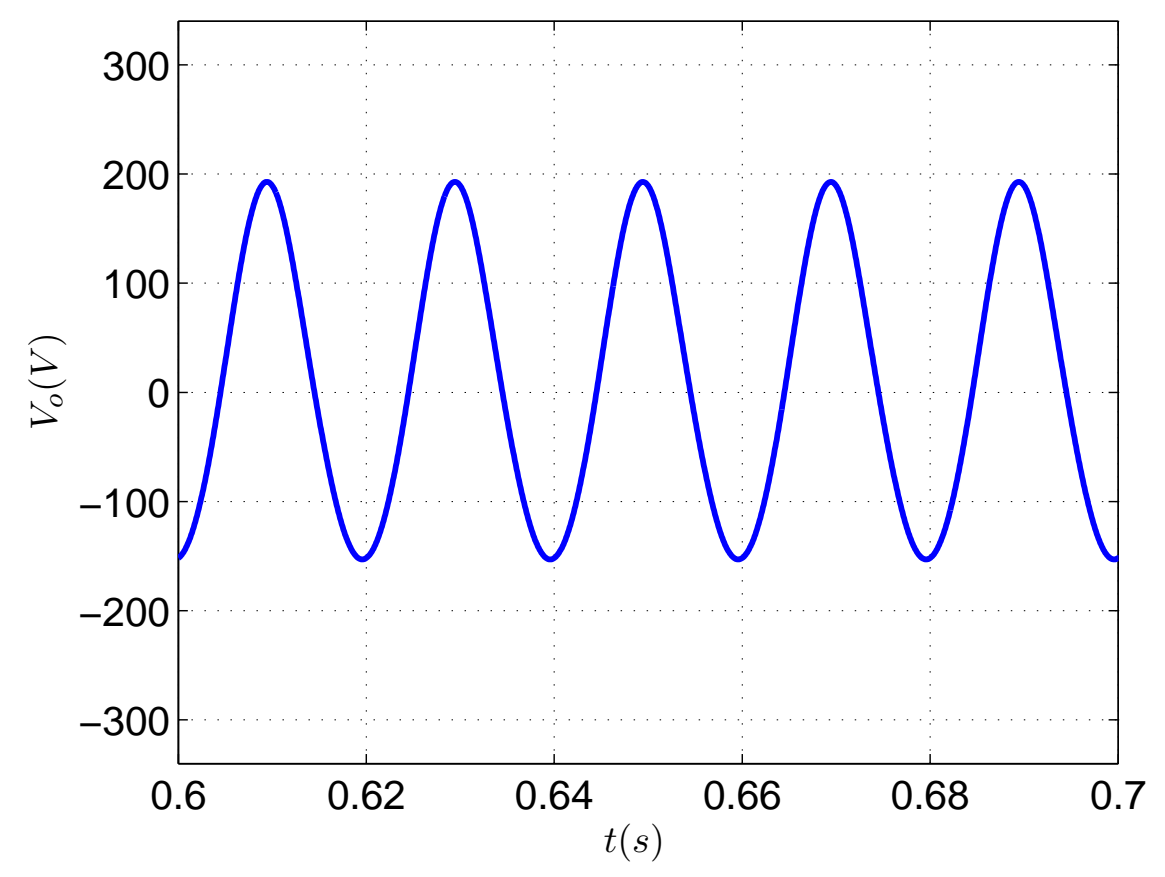

Figure 6: Output voltage of the boost inverter. 


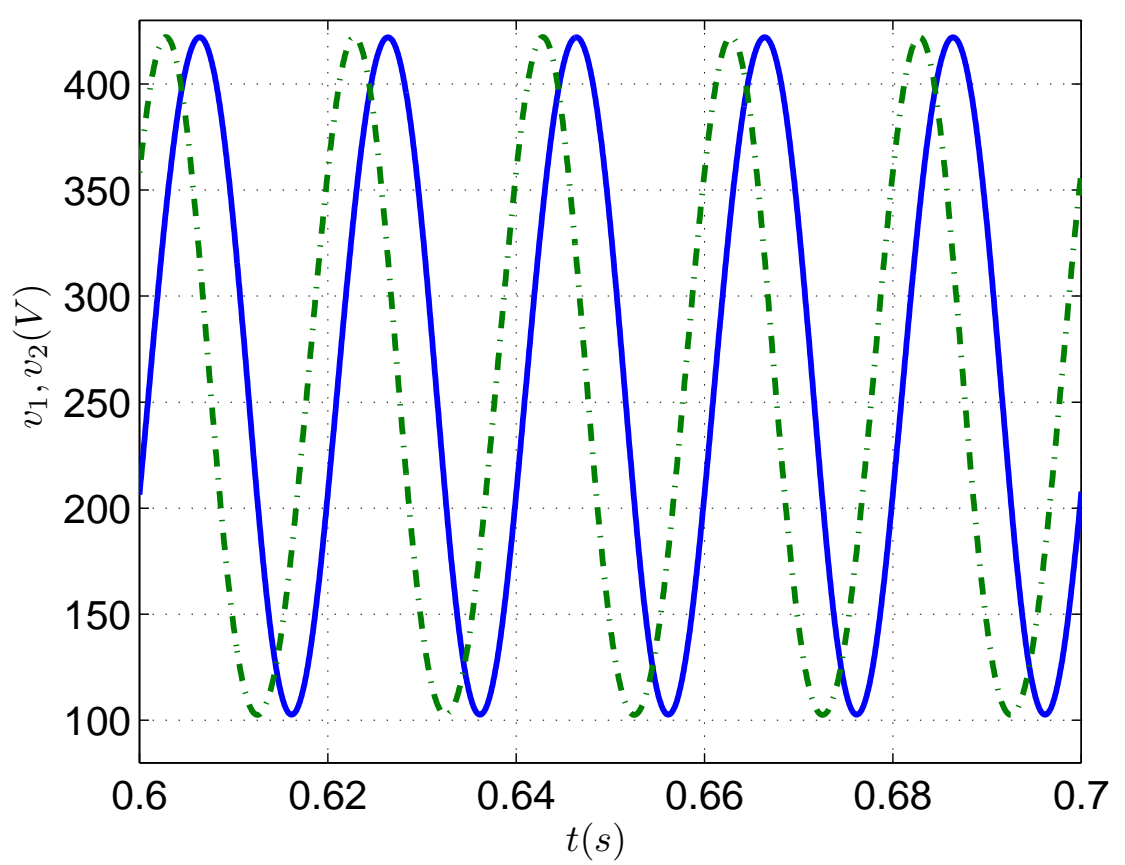

Figure 7: Output voltages of the first (solid) and second (dashed) boost DC-DC converters. 
controls a DC-DC converter. Therefore, in the above design, the voltage signal did not present the phase shift mentioned before. In order to get the desired output voltage, it is necessary to synchronize these signals: one with a phase shift of $180^{\circ}$, and the other one with a phase shift of $0^{\circ}$. In this section, a phase controller (PC), inspired by the configuration of a PhaseLock Loop (PLL) (Hsieh and Hung, 1996)-(Abramovitch, 2002), is added. The PC allows us to achieve the desired phase between the output of the two DC-DC converters as well as the synchronization of the boost inverter output with respect to a specified voltage signal, as in the case of synchronization with the electrical grid.

\subsection{Boost Inverter Synchronization}

The objective is to synchronize voltage signals $x_{2}$ and $x_{4}$ in anti-phase. The method is illustrated in Fig. 8. The normalized voltage of the second DC-DC converter, $x_{4}$, is taken as a reference signal and the normalized voltage of the first DC-DC converter, $x_{2}$, is the signal to be synchronized with $x_{4}$ in anti-phase. These are the inputs to the PC. The output is a frequency increment, which is added to the nominal frequency, $\omega$, in the Control 1 block and the resultant frequency is entered in (44). The output of the converter is a sinusoidal signal of that resulting frequency.

The PC block diagram appears in Fig. 9. The multiplier obtains the product, $x_{2}^{\prime} \times x_{4}^{\prime}$, in such way, (see Hsieh and Hung, 1996), that its output, once filtered by a low pass filter (LPF), is a measure of the deviation of the phase shift with respect to $90^{\circ}$. For this reason, one of the inputs of the multiplier, e.g. $x_{2}^{\prime}$, is obtained by passing voltage $x_{2}$ through a high pass filter (HPF) in order to eliminate its continuous component. Likewise, 


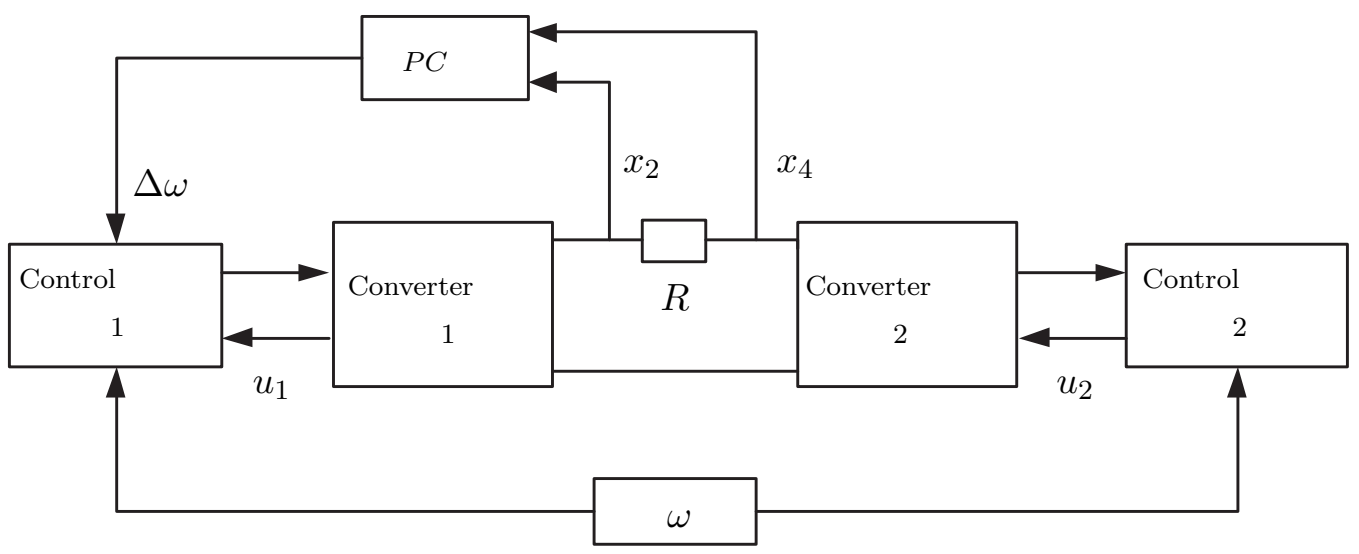

Figure 8: Block diagram of boost DC-AC converter with output voltages in anti-phase by the PC.

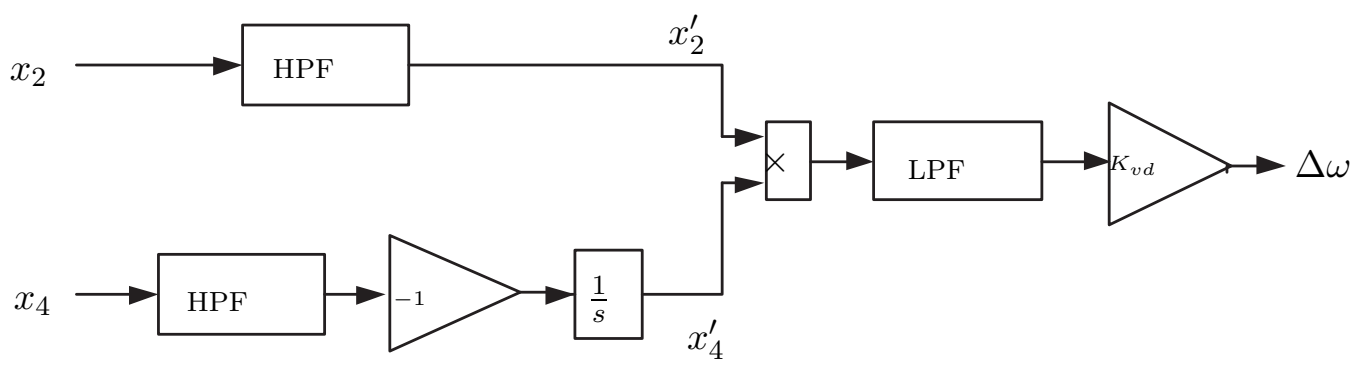

Figure 9: Conceptual block diagram of the PC.

$x_{4}^{\prime}$ is obtained after passing $x_{4}$ through another HPF and then changing its sign and integrating it. In this way, if $x_{4}=B-A \sin (\omega t)$, then, $x_{4}^{\prime}=$ $\frac{A}{\omega} \sin \left(\omega t-\frac{\pi}{2}\right)+C$. Constant $C$ is eliminated by the LPF.

Note that at state-stable $x_{2}=-x_{4}$ is forced by introducing the PC. For simplicity the PC in the stability analysis is not included. A rigorous analysis should take the PC into account. 
Simulation Results. The high pass filter applied is:

$$
\frac{1.4 s}{s+\omega}
$$

The LPF is a second order Butterworth filter (Ifeachor and Jervis, 2002):

$$
\frac{1}{\left(s+0.008\left(\frac{\sqrt{2}}{2}-\frac{\sqrt{2}}{2} j\right)\right)\left(s+0.008\left(\frac{\sqrt{2}}{2}+\frac{\sqrt{2}}{2} j\right)\right)} .
$$

The value of the PC gain is $K_{v d}=5 \cdot 10^{-4}$.

The results of the PC application are shown in Fig. 10. Voltages $v_{1}$ and $v_{2}$ in anti-phase. In Fig. 11 the boost inverter output voltage is represented.

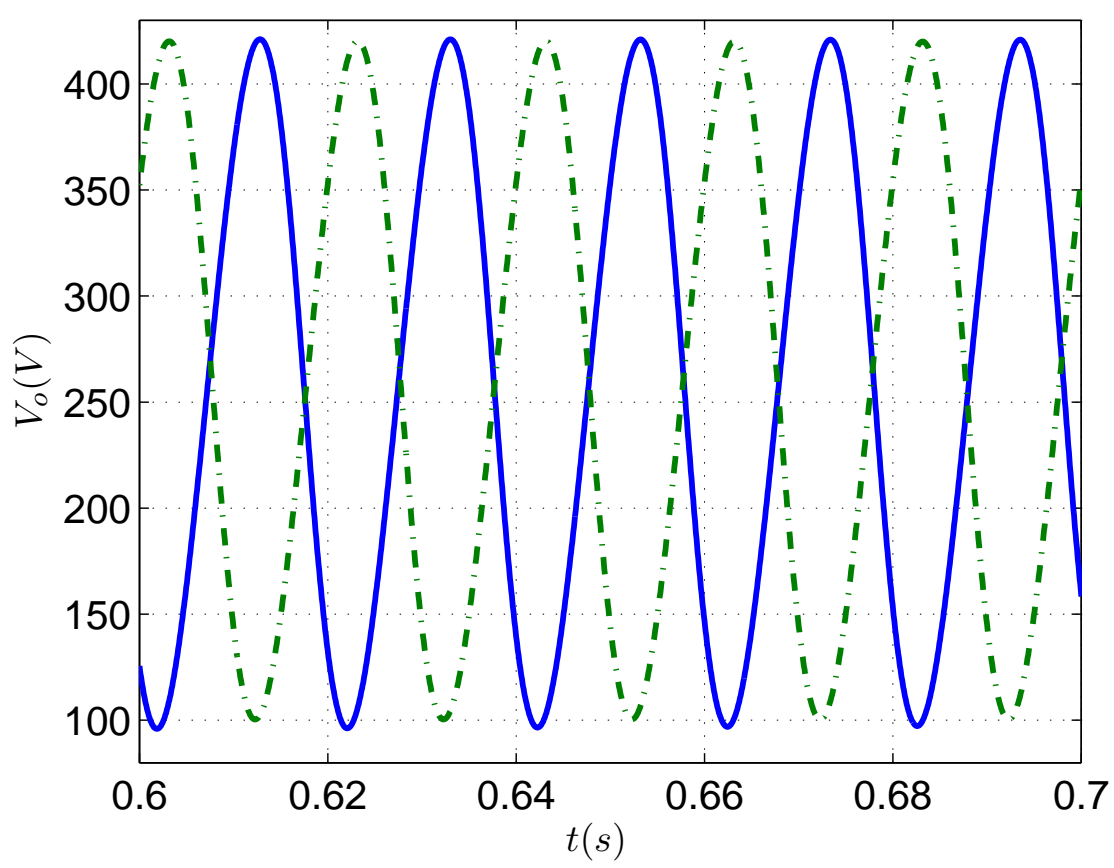

Figure 10: Output voltage of the first (solid) and second (dashed) boost DC-DC converters.

Figure 12 shows the ripple in the inductance currents, which is quite acceptable. 


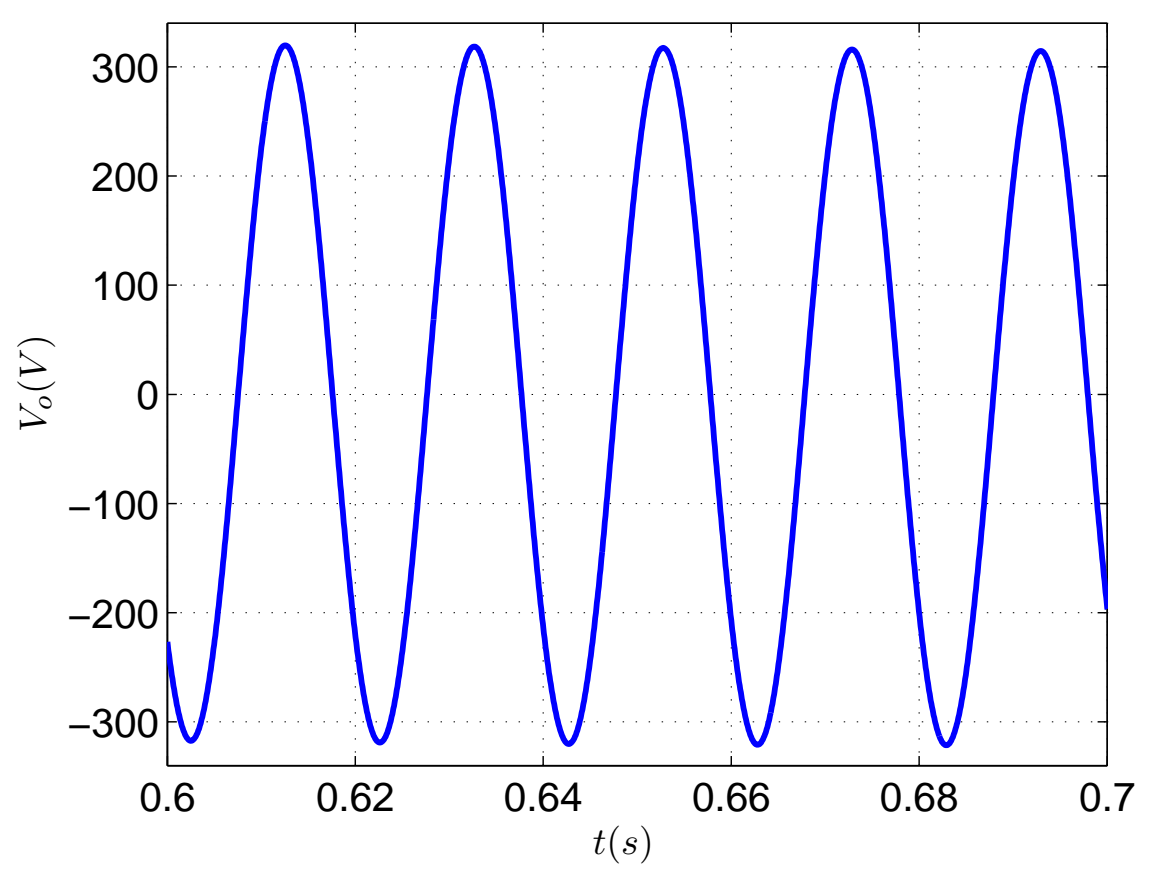

Figure 11: Output voltage with PC. 


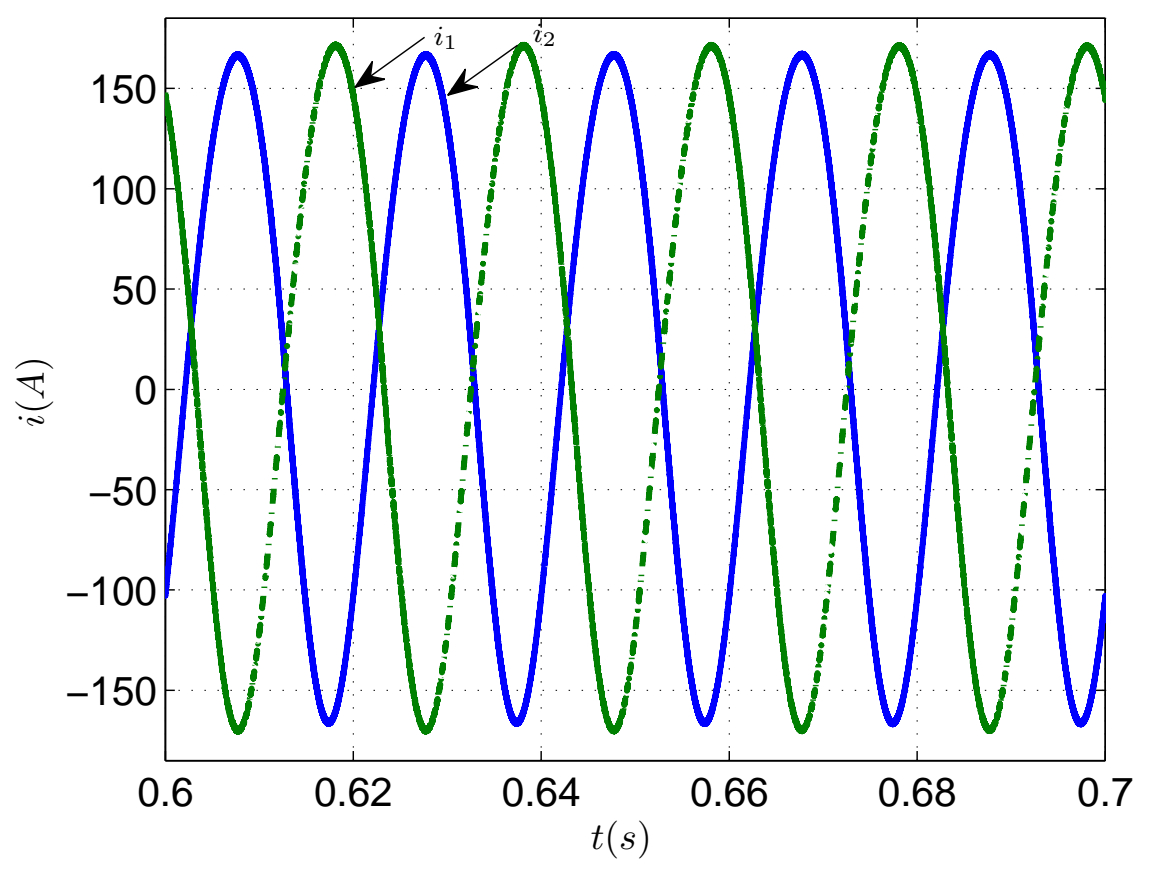

Figure 12: Inductance currents. 
The output signal has a $\mathrm{THD}=0.22 \%$. Figure 13 shows the signal spectrum of the signals $v_{2}$ and $v_{4}$. As can be seen, the harmonics of the fundamental frequency wave of the obtained output is quite satisfactory.

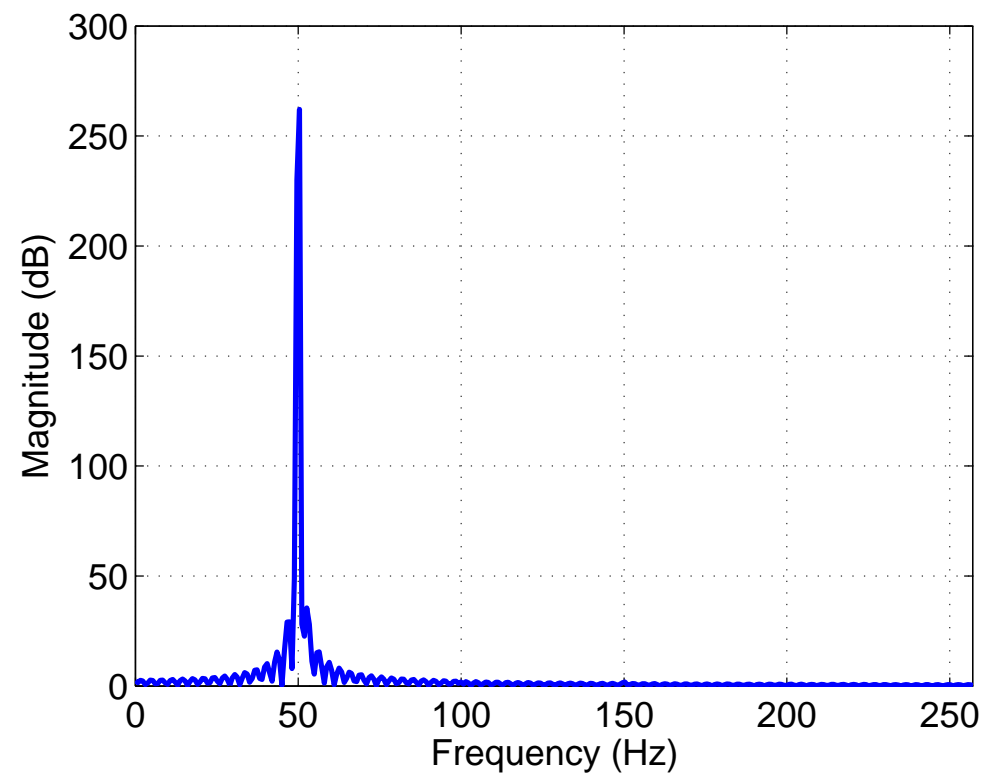

Figure 13: Output voltage signal spectrum.

This result justifies the first harmonic approximations carried out during the design of the control law. Of course, this is only valid for the chosen parameters and it does not prove the usefulness of the law in a general sense. In fact, the approximation does not work when the circuit parameters are not chosen adequately, but it is thought that, when the circuit is designed properly (taking into account the voltage, current and power levels), the approximations will yield good results. Note once more that these approximations are common in the literature (Fossas et al., 2008; Fossas and Olm-Miras, 2002). 


\subsection{Synchronization with the electrical grid}

In some applications, such as renewable energy plants, an inverter is necessary to inject energy from production plants into the electrical grid. In this case, the problem is synchronizing the voltage output with a pre-specified signal.

In order to synchronize the boost inverter output signal with the electrical grid, the normalized voltage signals of both DC-DC converters $\left(x_{2}, x_{4}\right)$ are treated with two PCs, as is shown in Fig. 14. $x_{2}$ is synchronized with the grid voltage using a phase shift of $180^{\circ}$ by using $\mathrm{PC} 1$, whose structure is shown in Fig. 15 and which is similar to the PC in Fig. 9. Signal $x_{4}$ is synchronized with the grid voltage using a zero phase shift by means of PC2 shown in Fig. 16. In this case, $g^{\prime}=\frac{A}{\omega} \sin \left(\omega t-\frac{\pi}{2}\right)$.

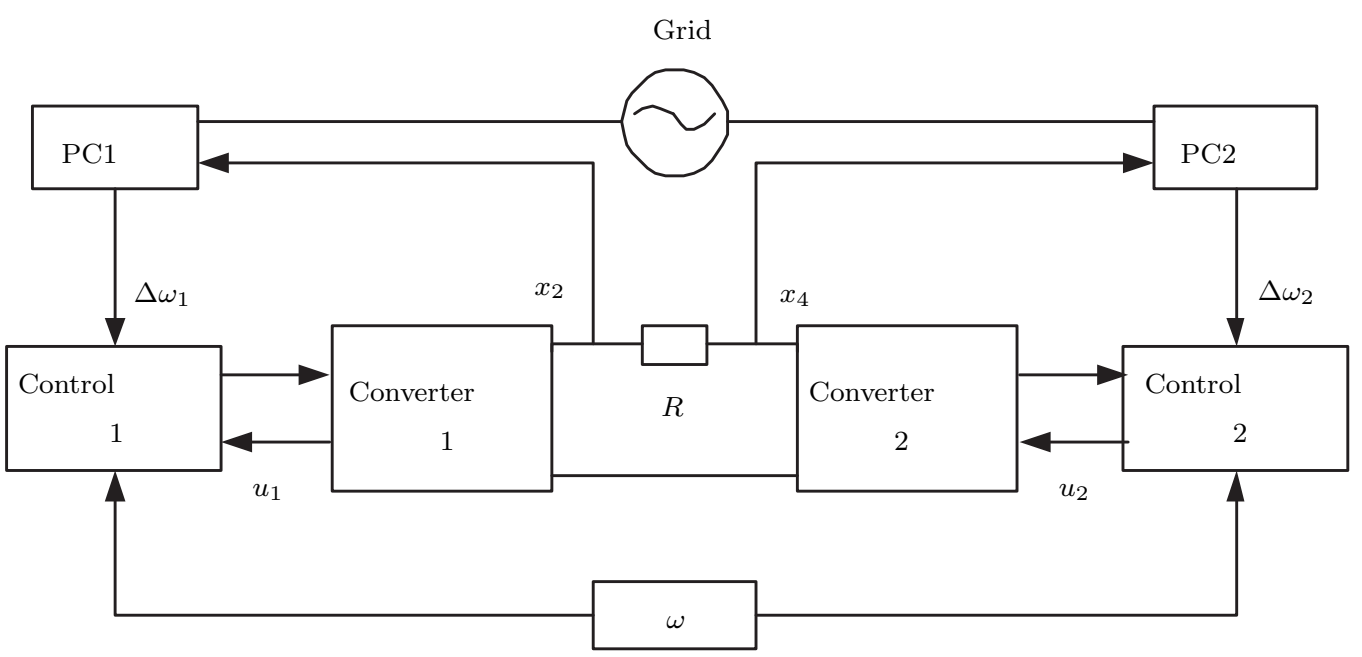

Figure 14: Block diagram of boost DC-AC converter with output voltage synchronized with the electrical grid. 


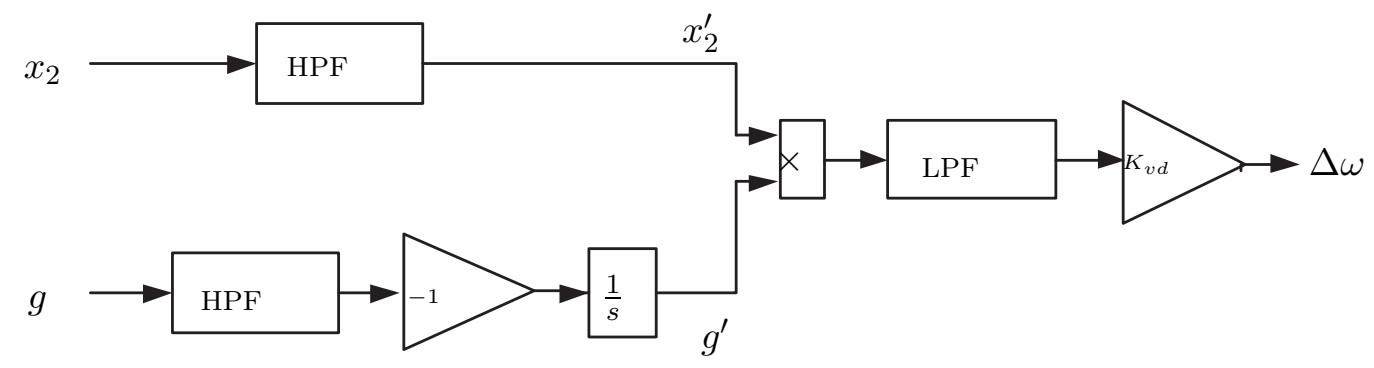

Figure 15: Conceptual block diagram of PC1.

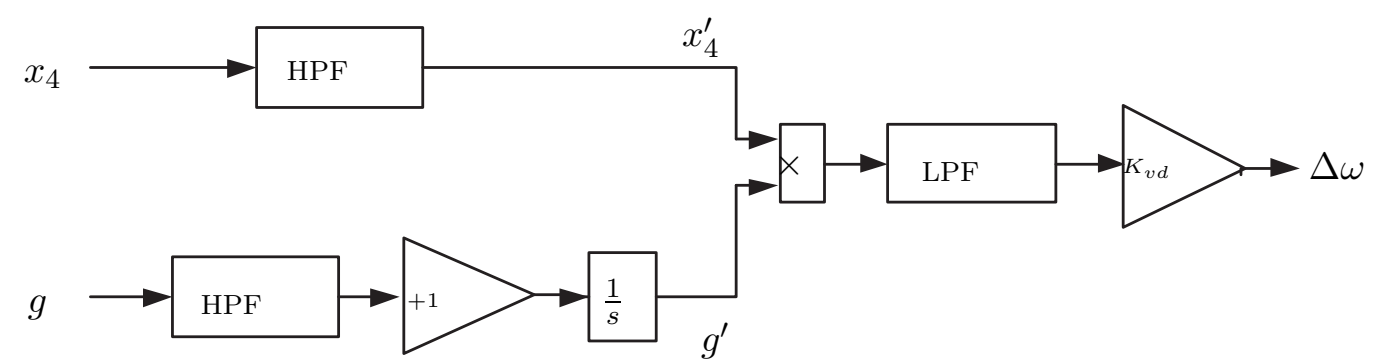

Figure 16: Conceptual block diagram of PC2. 
Simulation Results. The grid voltage is

$$
V_{\text {grid }}=220 \frac{2}{\sqrt{2}} \sin (100 \pi t)
$$

The values used in this simulation for the filter parameters and gain, $K_{v d}$, are the same ones used previously.

The performance of the synchronization of the boost DC-AC converter with the electrical supply voltage is represented in Fig.17.

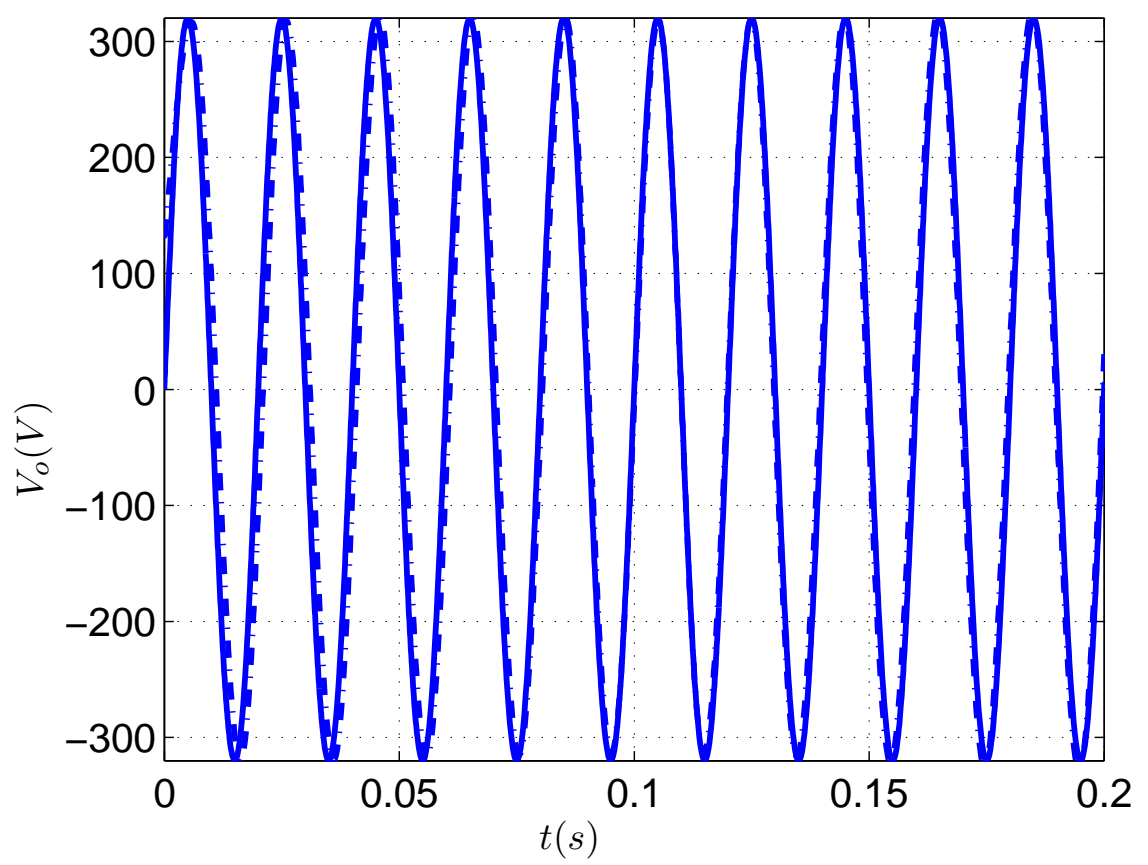

Figure 17: Electrical supply voltage (solid) and simulated output voltage synchronized with PCs (dashed). 


\section{Adaptive Control}

An adaptive law (or a load observer) is proposed to cope with load changes and/or load uncertainties. This observer is designed based only on a onesided circuit, which contains enough information to make this parameter observable. Therefore, the study of the full two-sided circuit is avoided due to symmetry considerations.

The observer design problem for one-sided circuit (left part of the Fig. 2) (10)-(11), can be rewritten compactly as:

$$
\begin{aligned}
\dot{x}_{l} & =U_{l} x_{l}+a D_{l} y+E_{l} \\
\dot{a} & =0 \\
y & =x_{2}-x_{4} \\
y_{m} & =M x_{l}
\end{aligned}
$$

with $x_{l}=\left[x_{1}, x_{2}\right]^{T} ; x_{4}$ can be considered as an input, and

$$
U_{l}=\left[\begin{array}{cc}
0 & -u_{1} \\
u_{1} & 0
\end{array}\right], D_{l}=\left[\begin{array}{c}
0 \\
-1
\end{array}\right], E_{l}=\left[\begin{array}{l}
1 \\
0
\end{array}\right], M=I_{2 \times 2} .
$$

Note, that $y \in \mathbb{R}^{1}$ and $y_{m} \in \mathbb{R}^{2}$.

Remark 1. In what follows, it is assumed that both voltage and current, i.e. $y_{m}$, are measurable, and thus accessible for control use

Remark 2. From Remark 1, note that $x_{l}$ and $y$ are measurable variables.

Furthermore, from Remark 1 and 2, it is clear that if both voltage and current are measurable, then it is evident the observability of system (50) does 
not depend on input, $u_{1}$. It is clear that a direct analysis of observability of the extended system, represented by $x=\left(x_{l}, a\right)$, is satisfied for $y$ different to 0. A phase controller can be designed such that the output $y$ becomes different to 0 . Therefore, these measured and generated signals are such that parameter $a$ becomes observable. The stability of the closed loop scheme will be guaranteed by using singular perturbation techniques.

\subsection{Adaptation law}

The proposed adaptation law is comprised of a state observer for one side of the inverter boost, plus an adaptation law for parameter $a$.

From Eq. (53): $K_{0}\left(y_{m}-\hat{y}_{m}\right)=K_{0}\left(M x_{l}-M \hat{x}_{l}\right)=K\left(x_{l}-\hat{x}_{l}\right)$ where $K_{0}, K \in \mathbb{R}^{2 \times 2}$ are constant design matrix.

Therefore, the adaptation law has the following structure:

$$
\begin{aligned}
\dot{\hat{x}}_{l} & =U_{l} x_{l}+\hat{a} D_{l} y+E_{l}+K\left(x_{l}-\hat{x}_{l}\right) \\
\dot{\hat{a}} & =\beta\left(x_{l}, \hat{x}_{l}\right),
\end{aligned}
$$

where $\beta\left(x_{l}, \hat{x}_{l}\right)$ is the adaptation law to be designed, $\hat{x}_{l}$ is the estimated state of $x_{l}$ and $\hat{a}$ is the estimated value of $a$. From Remark 2, the real state of $x_{l}$ and $y$ can be used in Eq. (54). Note that even if $x_{l}$ is accessible, the adaptation law designed here requires the additional (or extended) state observer. This will become clear during the analysis of the error equation system, as is shown below.

\subsection{Error equation}

Assume that $a$ is a constant parameter $(\dot{a}=0)$ or that it changes slowly $(\dot{a} \approx 0)$ and define the following error variables:

$$
\tilde{x}_{l}=x_{l}-\hat{x}_{l}, \quad \tilde{a}=a-\hat{a}, \quad \dot{\tilde{a}}=-\dot{\hat{a}} .
$$


Error equations are now derived from (50)-(53) together with (54)-(55)

$$
\begin{aligned}
\dot{\tilde{x}}_{l} & =-K \tilde{x}_{l}+\tilde{a} D_{l} y \\
\dot{\tilde{a}} & =-\beta\left(x_{l}, \hat{x}_{l}\right) .
\end{aligned}
$$

Let $K$ be of the form,

$$
K=\alpha I, \quad \alpha>0
$$

and $P=I$ be the trivial solution of $P K^{T}+K P=-Q$, with $Q=2 \alpha I$.

Now, if

$$
V=\tilde{x}_{l}^{T} P \tilde{x}_{l}+\frac{\tilde{a}^{2}}{\gamma}
$$

is introduced, it follows that

$$
\begin{aligned}
\dot{V} & =-\tilde{x}_{l}^{T} Q \tilde{x}_{l}+2 \tilde{a}\left(\tilde{x}_{l}^{T} P D_{l} y+\frac{\dot{\tilde{a}}}{\gamma}\right) \\
& =-\tilde{x}_{l}^{T} Q \tilde{x}_{l}+2 \tilde{a}\left(\tilde{x}_{l}^{T} P D_{l} y-\frac{\dot{\hat{a}}}{\gamma}\right) .
\end{aligned}
$$

The adaptation law is now designed by canceling the terms in square brackets, i.e.

$$
\dot{\hat{a}}=\gamma\left(D_{l}^{T} P \tilde{x}_{l}\right) y
$$

\subsection{Stability properties}

The observer and the adaptive law error equations are now fully defined. These equations are:

$$
\begin{aligned}
\dot{\tilde{x}}_{l} & =-K \tilde{x}_{l}+\tilde{a} D_{l} y \\
\dot{\tilde{a}} & =-\gamma\left(D_{l}^{T} P \tilde{x}_{l}\right) y .
\end{aligned}
$$


The stability properties of these equations follow from the Lyapunov function, $V$, defined above. Note that with choice (59) it follows that

$$
\dot{V}=-\tilde{x}_{l}^{T} Q \tilde{x}_{l}
$$

From standard Lyapunov arguments Khalil (2002), it follows that error variables $\tilde{x}_{l}$ and $\tilde{a}$ are bounded. In addition, according to the LaSalle invariant principle, it is easy to conclude that $\tilde{x}_{l} \rightarrow 0$, which implies from (61) that $\dot{\tilde{a}} \rightarrow 0$.

From (60), and from the property $\tilde{x}_{l} \rightarrow 0$, and $\dot{\tilde{x}}_{l} \rightarrow 0$, it is got

$$
\lim _{t \rightarrow \infty}\left\{\tilde{a}(t) D_{l} y(t)\right\}=0
$$

Note that if $y$ behaves as a sinusoidal, as is expected from the control problem formulation, the unique asymptotic solution for $\tilde{a}$, is $\tilde{a}=0$, as long as $y \not \equiv$ $0, \forall t \geq 0$.

The following lemma summarizes the above results, assuming that $y \not \equiv 0$ (the points where $y \equiv 0$ will be analyzed in the next section):

Lemma 1. Consider the open-loop system (50)-(53), and the observer (54)(55) with $K=\alpha I$ such that $K$ is a solution for $P K^{T}+K P=-Q$, then the observer states have the following properties:

i) The estimated states $\hat{x}_{l}$, $\hat{a}$ are bounded.

ii) $\lim _{t \rightarrow \infty} \hat{x}_{l}(t)=x(t)$.

iii) $\lim _{t \rightarrow \infty} \hat{a}(t)=a$, if $y(t) \not \equiv 0, \forall t \geq 0$. 


\section{Stability considerations of the full closed-loop system}

In the previous section, the stability properties were presented for the extended observer. These properties are independent of the evolution of the system state variables. The stability of the complete system is analyzed in this section.

The open-loop two-sided inverter (10)-(11) plus (42)-(43) normalized, can be compactly rewritten as:

$$
\begin{aligned}
& \dot{x}=U\left(u_{1}, u_{2}\right) x+a D y+E \\
& y=x_{2}-x_{4}
\end{aligned}
$$

with $x=\left[x_{1}, x_{2}, x_{3}, x_{4}\right]^{T}$, and

$$
U=\left[\begin{array}{cccc}
0 & -u_{1} & 0 & 0 \\
u_{1} & 0 & 0 & 0 \\
0 & 0 & 0 & -u_{2} \\
0 & 0 & u_{2} & 0
\end{array}\right], D=\left[\begin{array}{c}
0 \\
-1 \\
0 \\
1
\end{array}\right], E=\left[\begin{array}{l}
1 \\
0 \\
1 \\
0
\end{array}\right] .
$$

\subsection{Tuned System}

The tuned system is defined as the ideal closed-loop system controlled by the tuned feedback laws $u_{1}^{*}=\kappa_{1}\left(x, a^{*}\right)$ and $u_{2}^{*}=\kappa_{2}\left(x, a^{*}\right)$ (from Eq. (44) and (45), respectivaly), where $a^{*}$ is the exact value of $a$.

The tuned system given in (25)-(26) and (27) states

$$
\begin{aligned}
\dot{x} & =U\left(u_{1}^{*}, u_{2}^{*}\right) x+a D y+E \\
& =U\left(\kappa_{1}\left(x, a^{*}\right), \kappa_{2}\left(x, a^{*}\right)\right) x+a D y+E \\
& \triangleq f(x)
\end{aligned}
$$


and it achieves an asymptotically orbitally stable periodic solution, i.e.

$$
x^{*}(t)=x^{*}(t+T) .
$$

In Section 3, it was shown that functions $\Gamma_{1}$ and $\Gamma_{2}$ defined in (46)-(47) tend to zero. They correspond to periodic sinusoidal solutions of period $T=2 \pi / \omega$. Consequently, $y^{*}=x_{2}^{*}-x_{4}^{*}$ is also sinusoidal.

\subsection{Closed-loop system}

In practice, the control laws that are effectively applied depend on the estimation, $\hat{a}$, of parameter $a$. This control laws are denoted as $\hat{u}_{1}=\kappa_{1}(x, \hat{a})$ and $\hat{u}_{2}=\kappa_{2}(x, \hat{a})$, respectively. Note that these control laws depend on state $x$ and not on their estimations, $\hat{x}_{l}$, because state $x$ is directly measured. The role of $\hat{x}_{l}$ is then just to make the design of the adaptation law for $a$ possible.

The closed-loop equation resulting from the use of $\hat{u}_{1}=\kappa_{1}(x, \hat{a}), \hat{u}_{2}=$ $\kappa_{2}(x, \hat{a}), u_{1}^{*}=\kappa_{1}\left(x, a^{*}\right)$ and $u_{2}^{*}=\kappa_{2}\left(x, a^{*}\right)$ is written as

$$
\begin{aligned}
\dot{x} & =U\left(\hat{u}_{1}, \hat{u}_{2}\right) x+a D y+E+U\left(u_{1}^{*}, u_{2}^{*}\right) x-U\left(u_{1}^{*}, u_{2}^{*}\right) x \\
& =f(x)+\left[U\left(\hat{u}_{1}, \hat{u}_{2}\right)-U\left(u_{1}^{*}, u_{2}^{*}\right)\right] x
\end{aligned}
$$

Let us assume that $\hat{a} \in\left[\hat{a}_{m}, \hat{a}^{M}\right]$ and denote $\tilde{a}=a^{*}-\hat{a}$. Applying meanvalue theorem (Khalil, 2002) yields

$$
U\left(u^{*}\right)-U(\hat{u})=\mathcal{T}(x) \tilde{a},
$$

being

$$
\mathcal{T}(x) \triangleq\left[\begin{array}{cc}
\left.\mathbb{I} \frac{\partial u_{1}}{\partial a}\right|_{a=\hat{\bar{a}}} & 0 \\
0 & \left.\mathbb{I} \frac{\partial u_{2}}{\partial a}\right|_{a=\hat{\bar{a}}}
\end{array}\right]
$$


where, $\hat{\bar{a}}$ takes any value belonging to the interval $\mathcal{A} \triangleq\left[\min \left\{a^{*}, \hat{a}_{m}\right\}, \max \left\{a^{*}, \hat{a}_{M}\right\}\right]$, and

$$
\mathbb{I} \triangleq\left[\begin{array}{cc}
0 & -1 \\
1 & 0
\end{array}\right] .
$$

In view of the discussion above, this term has the following property:

Property 1. Let $\mathbb{M}=\left\{(x, \tilde{a}):|| x-x^{*}|| \leq \epsilon_{x},|\tilde{a}| \leq \epsilon_{a}\right\}$ be a compact domain that includes the asymptotic periodic solutions from the tuned system and the exact value of a e.i. $a^{*}$. Then, $\mathcal{T}(x) \tilde{a}$ has $\forall(x, \tilde{a}) \in \mathbb{M}$, the following properties:

i) it is continuous, analytic, and free of singularities

ii) $\lim _{\tilde{a} \rightarrow 0} \mathcal{T}(x) \tilde{a}=0$.

Putting (68) together with the observer error system yields the complete set of closed-loop equations, with $y=y(x)$

$$
\begin{aligned}
\dot{x} & =f(x)-\mathcal{T}(x) \tilde{a} x \\
\dot{\tilde{x}}_{l} & =-\alpha \tilde{x}_{l}+\tilde{a} D_{l} y \\
\dot{\tilde{a}} & =-\gamma\left(D_{l}^{T} P \tilde{x}_{l}\right) y
\end{aligned}
$$

where $K=\alpha I$. The stability consideration discussed here will be based on the time-scale separation. The main idea is that with the suitable choice of gains (as discussed below), observer equation (70)-(71) can be seen as the fast subsystem and equation (69) as the slow subsystem. Note again that this time-scale separation should be enforced by a particular choice of the observer and adaption gains $\alpha$ and $\gamma$. 
Note, that the manifold $y(t) \equiv 0$ has to be carefully analyzed since can cause problems (e.g. in Eq. 59). An intuitive explanation about the right system performance (as will be seen by simulation below) is the PC introduction, see Section 4. The PC objective is to achieve $x_{2}+x_{4}=0$, thus avoiding $y(t)=x_{2}-x_{4}=0$.

\subsection{Singular perturbation form}

To put the system above in the standard singular perturbation form, it is necessary to follow the next steps:

- introduce $\bar{a}=\frac{\tilde{a}}{\alpha}$,

- select $\gamma=\alpha^{2}$

- define $\varepsilon=\frac{1}{\alpha}$

With these considerations, it is obtained

$$
\begin{aligned}
\dot{x} & =f(x)-\mathcal{T}(x) \bar{a} x \\
\varepsilon \dot{\tilde{x}}_{l} & =-\tilde{x}_{l}+\bar{a} D_{l} y \\
\varepsilon \dot{\bar{a}} & =-\left(D_{l}^{T} P \tilde{x}_{l}\right) y
\end{aligned}
$$

where $\varepsilon>0$ is the small parameter. Note that this particular selection of gains imposes relative gains for the adaptation, $\gamma$, and defines precisely how the observer gain is related to $\gamma$. If the target system (22)-(23) is divided by $\omega^{2}$, then its ends up with a similar form to the fast variables equations (70)-(71). 
Remark 3. The perturbed variable parameter, $\varepsilon=\varsigma(\tilde{a})$, and for a side effect, the adaptation gain, $\gamma$, must fulfill

$$
\begin{gathered}
\varepsilon \ll \min \left\{\frac{1}{\omega}, \frac{1}{k}\right\} \\
\gamma \gg \max \left\{\omega^{2}, k^{2}\right\} .
\end{gathered}
$$

These relationships with respect to the tuning parameter, $k$, and desired frequency, $\omega$, ensures the convergency of the observer and adaptation parameter, $a$.

Letting $z=\left[\tilde{x}_{l}, \bar{a}\right]^{T}$ yields the general form

$$
\begin{aligned}
\dot{x} & =f(x)-\mathcal{T}(x) \bar{a} x \\
\varepsilon \dot{z} & =g(x, z)
\end{aligned}
$$

with $x\left(t_{0}\right)=x^{0}, x \in \mathbb{R}^{4}, z\left(t_{0}\right)=z^{0}, z \in \mathbb{R}^{3}$, and

$$
g(z, x)=\left[\begin{array}{c}
-\tilde{x}_{l}+\bar{a} D_{l} y \\
-\left(D_{l}^{T} P \tilde{x}_{l}\right) y
\end{array}\right]
$$

According to the singular perturbation analysis, the next steps must be followed:

1. Find a stationary solution of the fast subsystem (73) by finding the roots of the equation $g(x, z)=0$, i.e. $z=\phi(x)$.

2. Replace this solution in the slow subsystem (72), and find the resulting slow system

$$
\dot{x}=f(x)-\mathcal{T}(x) \phi(x) x .
$$

3. Check the boundary layer properties of the fast subsystem along one particular solution of $\dot{x}=f(x)-\mathcal{T}(x) \phi(x) x$. 


\subsection{Slow sub-system}

Proceeding with steps 1 and 2 above requires that the solution for the algebraic equation for $g(x, z)=0$, whose roots are calculated from, is found:

$$
\begin{aligned}
\tilde{x}_{l} & =\bar{a} D_{l} y \\
0 & =-\bar{a} D_{l}^{T} P D_{l} y^{2} .
\end{aligned}
$$

Note that $D_{l}^{T} P D_{l}=2$, and that the above equation has multiple solutions, i.e

$$
\begin{aligned}
\tilde{x}_{l} & =0 \\
\bar{a} y^{2}(x) & =0
\end{aligned}
$$

which means that if $y \equiv 0$, there is one solution for $\tilde{x}_{l}=0$, and infinite solutions for $\bar{a}$. However, if $y \not \equiv 0$, for instance, in the particular tuned solution $y^{*}=A \sin (\omega t)$, then

$$
z=\phi(x)=\left[\begin{array}{c}
\tilde{x}_{l} \\
\bar{a}
\end{array}\right]=0
$$

becomes an isolated root. Thus, for this particular solution, and taking into account that $\bar{a}=\frac{a-\hat{a}}{\alpha}=0$, i.e. $\hat{a}=a$, the slow model is written as:

$$
\dot{x}=f(x)-\mathcal{T}(x) \cdot 0 \cdot x=f(x),
$$

which is nothing other than the tuned system whose solutions $x(t)=x^{*}(t)$ are sinusoidal.

\subsection{Boundary layer fast subsystem}

The next step is to evaluate the stability of the boundary layer system in the finite time interval $t \in\left[t_{0}, t_{1}\right]$. This is obtained by evaluating the fast 
subsystem (73) along one particular solution of the quasi-steady-state $x_{p}(t)$, and by re-scaling time $t$ to the stretched time coordinates $\tau=\left(t-t_{0}\right) / \varepsilon$. The fast subsystem (73) evaluated along this trajectory is

$$
\begin{aligned}
\frac{d}{d \tau} \hat{\tilde{x}}_{l_{1}} & =-\hat{\tilde{x}}_{l_{1}} \\
\frac{d}{d \tau} \hat{\tilde{x}}_{l_{2}} & =-\hat{\tilde{x}}_{l_{2}}-\hat{\bar{a}} y_{p} \\
\frac{d}{d \tau} \hat{\bar{a}} & =\hat{\tilde{x}}_{l_{2}} y_{p},
\end{aligned}
$$

which can be rewritten as:

$$
\frac{d}{d \tau} \hat{z}=J\left(y_{p}\right) \hat{z}=J(\tau, \omega, \varepsilon) \hat{z}
$$

with

$$
J=\left[\begin{array}{ccc}
-1 & 0 & 0 \\
0 & -1 & -y_{p} \\
0 & y_{p} & 0
\end{array}\right]
$$

Under these conditions, system (75) is reduced to the autonomous linear system

$$
\frac{d}{d \tau} \hat{z}=J(\tau, \omega, 0) \hat{z}=J\left(y_{p_{0}}\right) \hat{z}
$$

Consider the $y_{p_{0}} \in \mathcal{D}_{x}$, with $\mathcal{D}_{x} \triangleq\left\{x:|y|=\left|x_{2}-x_{4}\right|>\epsilon_{0}\right\}$. The above system has the following properties.

Property 2. The eigenvalues of $J\left(y_{p}\right)$, for $\left[t, x_{p}, z\right] \in\left[t_{0}, t_{1}\right] \times D_{x} \times \mathbb{R}^{3}$, are all strictly negative, i.e.

$$
\begin{aligned}
& \lambda_{1}=-1 \\
& \left.\lambda_{2}=\mathfrak{R e}\left\{\frac{\left.-1+\sqrt{1-4 y_{p}^{2}}\right\}<0}{2}\right\}<\frac{-1-\sqrt{1-4 y_{p}^{2}}}{2}\right\}<0, \\
& \lambda_{3}=\mathfrak{R e}\left\{\frac{-1}{2}\right\}
\end{aligned}
$$


where $\epsilon_{0}>0$ is a constant.

Therefore $J\left(y_{p}\right)$ is Hurwitz in the considered domain. As a result, there exists a matrix $P\left(y_{p}\right)=P\left(y_{p}\right)^{T}>0$ and a $Q\left(y_{p}\right)>0$ such that the standard Lyapunov equation holds:

$$
P\left(y_{p}\right) J\left(y_{p}\right)+J\left(y_{p}\right)^{T} P\left(y_{p}\right)=-Q\left(y_{p}\right) .
$$

From standard Lyapunov arguments, it follows that for all $t \in\left[t_{0}, t_{1}\right]$,

$$
\|\hat{z}(t, \varepsilon)\| \leq c_{1} \exp \left\{-\lambda_{\min }\left(Q\left(y_{p}\right)\right)\left(\frac{t-t_{0}}{\varepsilon}\right)\right\}
$$

Tikhonov's theorem (see Khalil, 2002) can now be used to summarize the previous result.

Theorem 1. There exists a positive constant $\varepsilon^{*}$ such that for all $y_{p_{0}} \in \mathcal{D}_{x}$, and $0<\varepsilon<\varepsilon^{*}$, the singular perturbation problem of (72)-(73) has a unique solution, $x(t, \varepsilon), z(t, \varepsilon)$ on $\left[t_{0}, t_{1}\right]$, and

$$
\begin{aligned}
x(t, \varepsilon)-x_{p}(t) & =O(\varepsilon) \\
z(t, \varepsilon)-\hat{z}_{p}(t / \varepsilon) & =O(\varepsilon)
\end{aligned}
$$

hold uniformly for $t \in\left[t_{0}, t_{1}\right]$, where $\hat{z}_{p}(\tau)$ is the solution of the boundary layer model (77). Moreover, given any $t_{b}>t_{0}$, there is $\varepsilon^{* *} \leq \varepsilon^{*}$ such that

$$
z(t, \varepsilon)=O(\varepsilon)
$$

holds uniformly for $t \in\left[t_{b}, t_{1}\right]$ whenever, $\varepsilon<\varepsilon^{* *}$.

In order to extend this result to an infinite time interval, it is necessary that the boundary layer system is exponentially stable in a neighborhood of the 
tuned slow solution $x_{p}(t)$ for all $t \geq t_{0}$. This may not be a simple proof, and it will be left for further investigation. Instead, the effectiveness of this approach is shown below using simulation.

An intuitive yet not completely rigorous explanation for the resulting good behavior in the infinite time interval can be given with the help of Fig. 18. Notice that the Hurwitz nature of Jacobian (76) is only lost when $y=x_{2}-x_{4}=0$. Since the fast motion, $z$, evolves with almost constant $y$ (vertical lines in Fig. 18), $y$ will not reach the value zero during this motion provided that $y$ is initially far enough from zero. Once the slow manifold is reached, the slow variable will evolve in the domain $z=0$. This domain corresponds to the case when the adaptation mechanism has reached its objective and parameter $a$ is correctly estimated. In this domain $y$ may reach the value zero but, intuitively, it is assumed that the system, once the adaptation law has reached the correct value, will present a behavior that is similar to the known load case, whose stability is proved in Section 3.

\subsection{Simulations}

The inverter parameter values are the same as those in the known load case given in the Section 3, where the load resistance was $50 \Omega$ and, therefore, $a=0.01$. The desired output is

$$
V_{o}=220 \frac{2}{\sqrt{2}} \sin (100 \pi t)
$$

At time $t=0 s$, the chosen value for the adaptation parameter is $\hat{a}=0.001$ (which corresponds to $R_{0}=500 \Omega$, i.e. the relative error is $90 \%$ ). Later, at time $t=1.2 s$, a load variation is produced from $R=50 \Omega$ to $R=500 \Omega$, so parameter $a$ is again equal to 0.01 


$$
0
$$


Once again, a commutation time of $50 \mathrm{KHz}$ is employed as is a sample time of $0.1 T \mathrm{~s}$.

Figures 19 and 20 show the evolution of the output voltage and the voltages of every boost DC-DC in $t=0 s$, respectively. Note that the circuit tends to the desired behavior. During this period the adaptation mechanism does not destabilize the system.

Figures 21 and 22 show the evolution of the output voltage and the voltages of every boost DC-DC in $t=1.2 s$, respectively. Note that the circuit achieves the desired behavior. The time scale is the real time scale before the change of variable. Note that during this time, when the perturbation in the resistance and, thus, the corresponding adaptation mechanism is activated, the output signal does not undergo a significant variation.

The adaptation of parameter $a$ is represented in Figs. 23 and 24 where the load-change instants in the transition and steady-state are zoomed respectively. Note that the adaptation is very fast relative to the time scale of the system. In each of these graphs two evolutions are presented for two different values of $\varepsilon$. Note the smaller $\varepsilon$ is, the faster the adaptation is.

\section{Conclusions}

A control strategy for the nonlinear boost inverter was presented. The method is based on energy-shaping methodology, which generates a limit cycle without requiring external reference signals. The resulting controller achieves the objective by adding a phase controller. The same idea is used in order to solve the problem of grid electrical synchronization.

After these results, a common and important problem is dealt with in the 


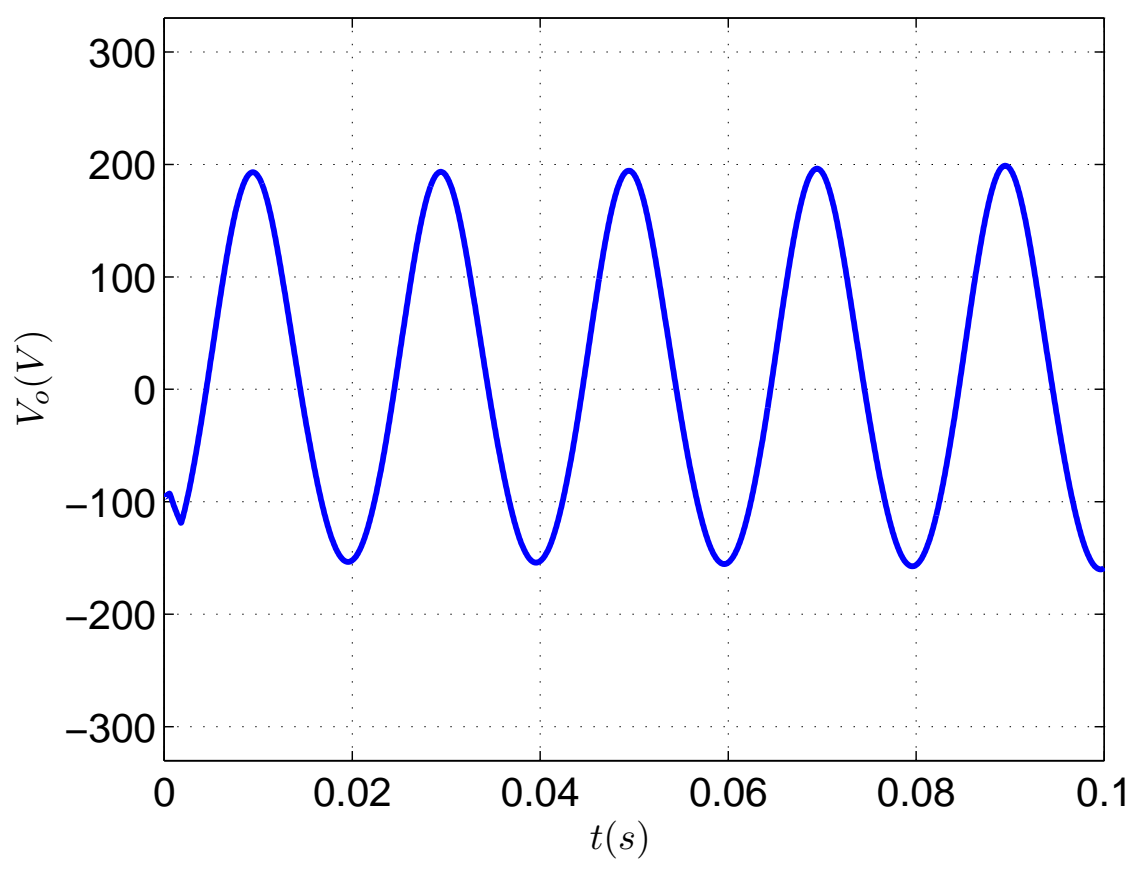

Figure 19: Output voltage with the adaptation of an unknown load in $t=0 \mathrm{~s}$. 


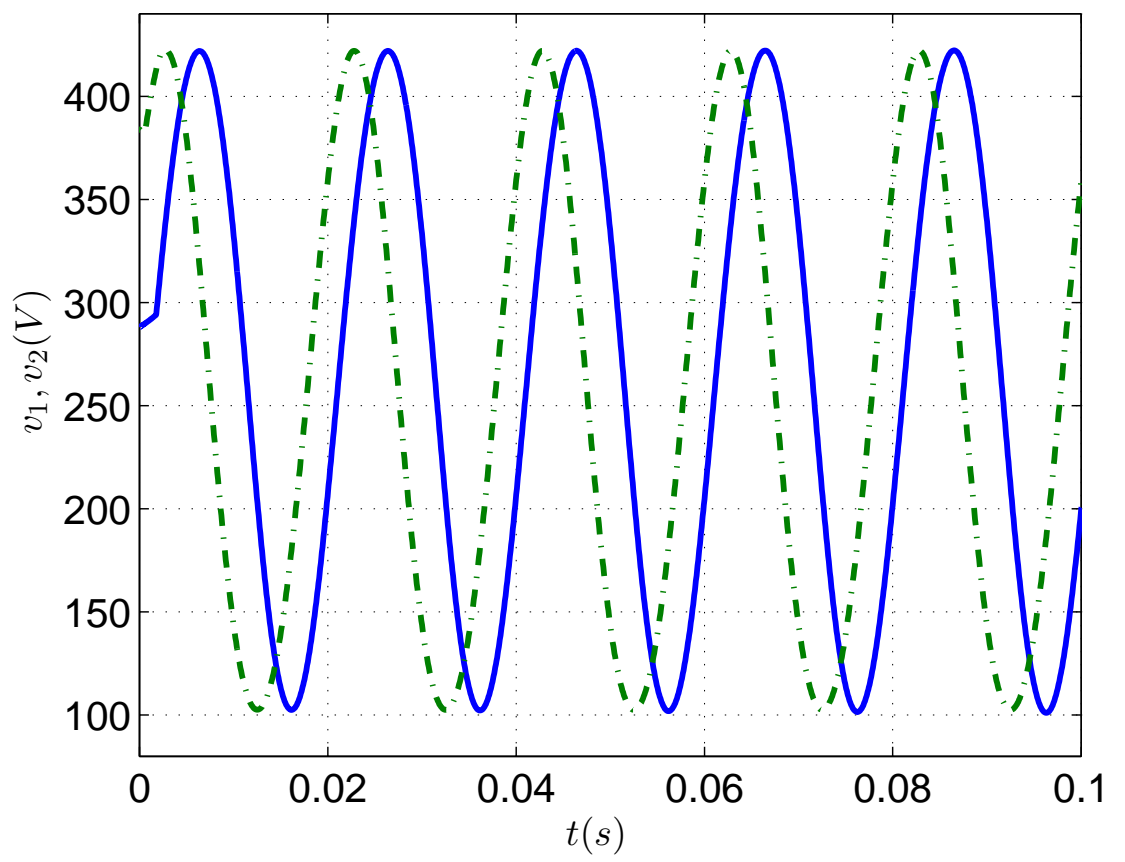

Figure 20: Output voltages of the first (solid) and second (dashed) boost DC-DC converters with the adaptation for an unknown load in $t=1.2 \mathrm{~s}$. 


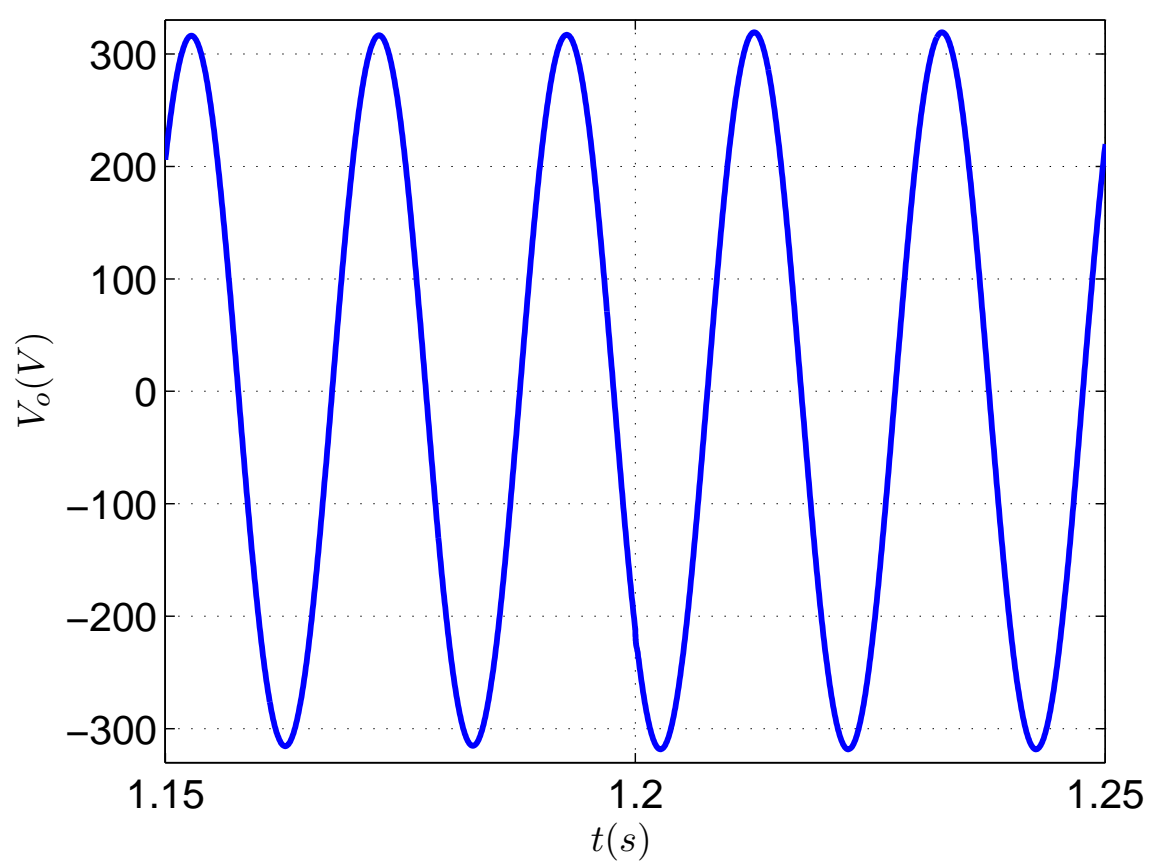

Figure 21: Output voltage with the adaptation of an unknown load in $t=0 \mathrm{~s}$. 


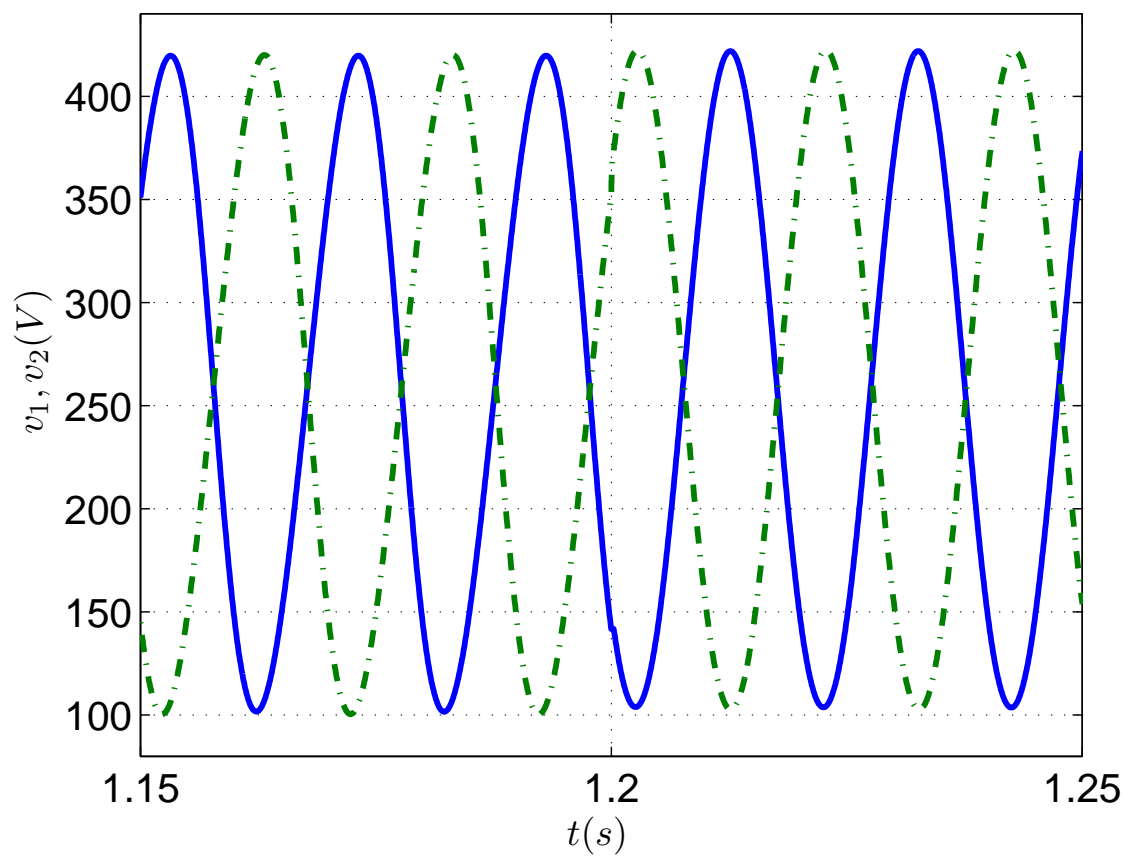

Figure 22: Output voltages of the first (solid) and second (dashed) boost DC-DC converters with the adaptation for an unknown load in $t=1.2 \mathrm{~s}$. 

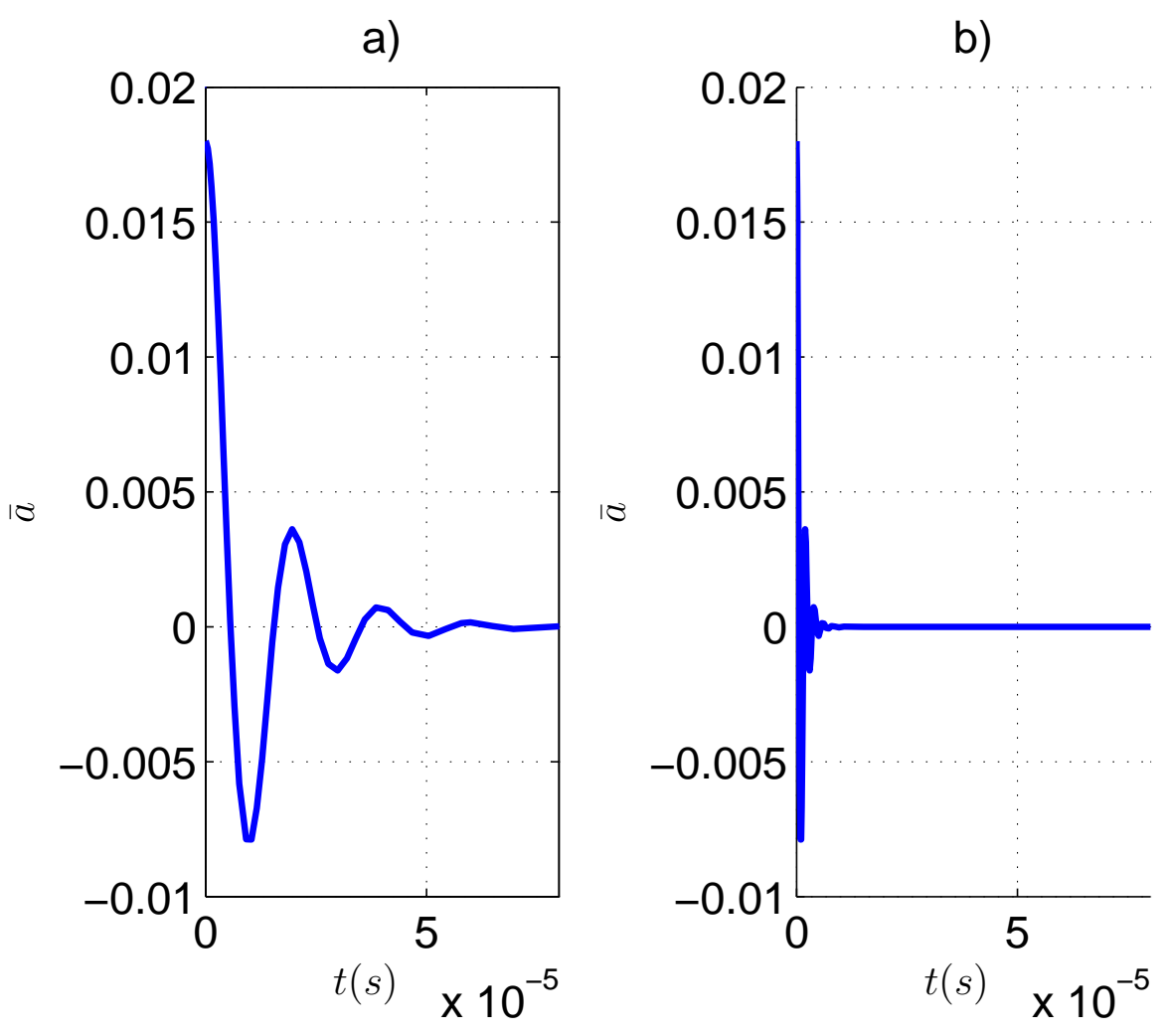

Figure 23: Time-evolution of the fast variable $\bar{a}$ with $\varepsilon=0.01$ in $a$ ) and $\varepsilon=0.001$ in $b$ ). The perturbation is at $t=0 \mathrm{~s}$. 

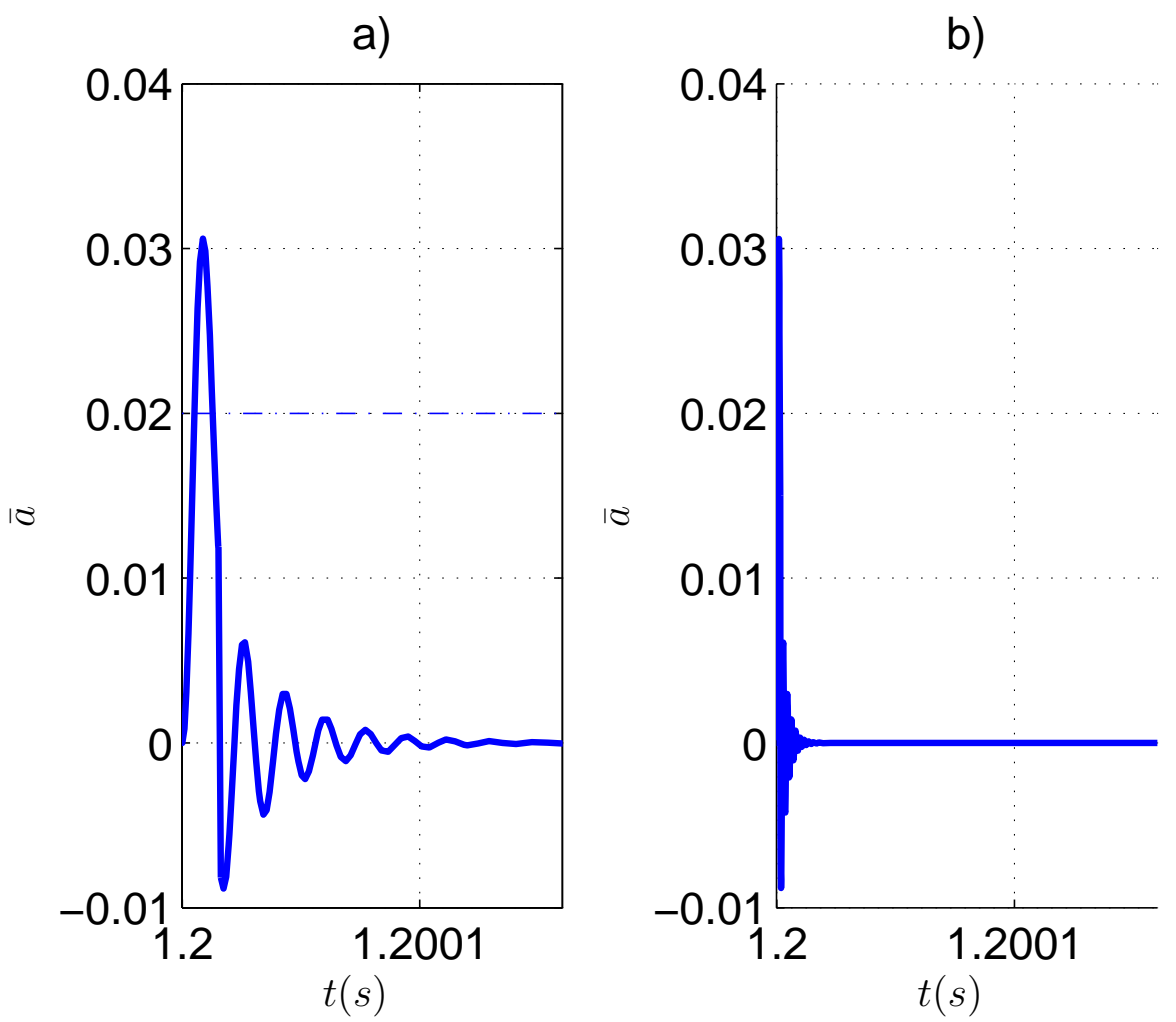

Figure 24: Time-evolution of the fast variable $\bar{a}$ with $\varepsilon=0.01$ in $c$ ) and $\varepsilon=0.001$ in $d$ ). The perturbation is at $t=0.5 \mathrm{~s}$. 
field of electronics: the load is unknown. For that, an adaptive control for unknown load is developed, which adapts the load very fast with respect to the time evolution of the system. The method is based on using a state observer on one-sided inverter and assuming that the state variables are measured.

The stability of the complete system is proved by putting the system in the standard singular perturbation form; hence some relationship between the adaptation gain, $\gamma$, the observer matrix parameter, $\alpha$, and the perturbed variable parameter, $\varepsilon$ are achieved. Another important relationship between the perturbed variable parameter, $\varepsilon$, and the system frequency, $\omega$, is achieved in the analysis of the boundary layer fast subsystem. Finally, the stability is established by means of Tikhonov's theorem (Khalil, 2002).

The assumption that voltage and current are measurable simplifies the observation problem. No persistant signals are required to prove the stability and the no noise is included in the measurable signals. As future work, an extension of this development could be done assuming that only the currents are measurable and including experimental results in order to validate all assumptions established in the paper.

\section{Acknowledgment}

The authors appreciate the valuable comments by the anonymous reviewers of this paper.

\section{References}

Abramovitch, D. (2002). Phase-Locked Loops: a control centric tutorial. in Proc. American Control Conference (ACC), 1, $1-15$. 
Albea, C., Canudas-de Wit, C., and Gordillo, F. (2007). Adaptive control of the boost dc-ac converter. in Proc. IEEE Conference on Control Applications (CCA), 611-616.

Albea, C. and Gordillo (2007). Estimation of the Region of Attraction for a Boost DC-AC Converter Control Law. in Proc IFAC Symposium on Nonlinear Control Systems (Nolcos), 874-879.

Albea, C., Gordillo, F., and Aracil, J. (2006). Control of the Boost DCAC Converter by Energy Shaping. in Proc. IEEE Industrial Electronics (IECON), 754-759.

Alonso, J., Ribas, J., Coz, J., Calleja, A., Corominas, E., and Rico-Secades, M. (2000). Development of a distributive control scheme for fluorescent lighting based on LonWorks technology. IEEE Transactions on Industrial Electronics, 47(6), 1253-1262.

Aracil, J. and Gordillo, F. (2002). On the control of oscillations in DC-AC converters. in Proc. IEEE Industrial Electronics (IECON), 4, 2820 - 2825.

Biel, D., Fossas, E., Guinjoan, F., Alarcón, E., and Poveda, A. (2001). Application of sliding-mode control to the design of a buck-basedsinusoidal generator. IEEE Transactions on Industrial Electronics, 48(3), 563-571.

Biel, D., Guinjoan, F., Fossas, E., and Chavarría, J. (2004). Sliding-mode control design of a boost-buck switching converter for AC signal generation. IEEE Trans. Circuits and Systems I: Regular Papers, 51(8), 1539-1551.

Cáceres, R. and Barbi, I. (1999). A boost DC-AC converter: analysis, design, and experimentation. IEEE Trans. Power Electronics, 14(1), 134-141. 
Carrasco, J., Quero, J., Ridao, F., Perales, M., and Franquelo, L. (1997). Sliding mode control of a DC/DC PWM converter with PFC implementedby neural networks. IEEE Trans. Circuits and Systems I: Fundamental Theory and Applications, 44(8), 743-749.

de Souza, K., de Castro, M., and Antunes, F. (2000). A DC/AC converter for single-phase grid-connected photovoltaic systems. 4, $3268-3273$.

Dixon, J. and Ooi, B. (1988). Indirect current control of a unity power factor sinusoidal currentboost type three-phase rectifier. IEEE Transactions on industrial electronics, 35(4), 508-515.

Elmas, C., Deperlioglu, O., and Sayan, H. (2007). Adaptive fuzzy logic controller for DC-DC converters. Expert Systems With Applications.

Fossas, E., Olm, J., and Sira-Ramírez, H. (2008). Iterative approximation of limit cycles for a class of Abel equations. Physica D: Nonlinear Phenomena, $237(23), 3159-3164$.

Fossas, E. and Olm-Miras, J. (2002). Asymptotic tracking in DC-to-DC nonlinear power converters. Discrete And Continuous Dynamical Systems Series B, 2(2), 295-311.

Gómez-Estern, F., Aracil, J., and Gordillo, F. (2002). The Hopf bifurcation and controlled oscillations in electromechanical systems. in Proc. Mediterranean Conference on Control and Automation (MED).

Gordillo, F., Aracil, J., and Gómez-Estern, F. (2002). Stabilization of autonomous oscillations and the Hopf bifurcation in the ball and beam. in Proc. IEEE Decision and Control (CDC), 4, 3924 - 3925. 
Gordillo, F., Pagano, D., and Aracil, J. (2004). Autonomous oscillation generation in electronic converters. in Proc. IEEE International Workshop on Electronics and System Analysis (IWESA).

Griño, R., Fossas, E., and Biel, D. (2002). Sliding mode control of a fullbridge unity power factor rectifier. Lecture Notes in Control and Information Sciences, 139-148.

H., S.R., Tarantino-Alvarado, R., and Llanes-Santiago, O. (1993). Adaptive feedback stabilization in PWM-controlled DC-to-DC power supplies. International Journal of Control, 57(3), 599-625.

Hadri-Hamida, A., Allag, A., Hammoudi, M., Mimoune, S., Zerouali, S., Ayad, M., Becherif, M., Miliani, E., and Miraoui, A. (2008). A nonlinear adaptive backstepping approach applied to a three phase PWM AC-DC converter feeding induction heating. Communications in Nonlinear Science and Numerical Simulation.

Hsieh, G. and Hung, J. (1996). Phase-Locked Loop techniques. A survey. IEEE Trans. Industrial Electronics, 43(6), 609-615.

Ifeachor, E. and Jervis, B. (2002). Digital Signal Processing: A Practical Approach. Pearson Education.

Khalil, H.K. (2002). Nonlinear Systems. Prentice Hall, third edition edition.

Kokotovic, P., Khalil, H.K., and O'Reilly, J. (1999). Singular Perturbation Methods in control Analysis and Design. SIAM. 
Malinowski, M., Marques, G., Cichowlas, M., and Kazmierkowski, M. (2003). New direct power control of three-phase PWM boost rectifiers under distorted and imbalanced line voltage conditions. In IEEE International Symposium on Industrial Electronics, (ISIE), volume 1.

MeVay, A. and Sarpeshkar, R. (2003). Predictive comparators with adaptive control. IEEE Trans. Circuits and Systems II: Analog and Digital Signal Processing, 50(9), 579-588.

Middlebrook, R. and Ćuk, S. (1977). A general unified approach to modelling switching-converter power stages. International Journal of Electronics, $42(6), 521-550$.

Ng, T., Leung, F., and Tam, P. (1996). A simple adaptive control strategy for regulated switching DC-DC converter based on grid-point concept. in Proc. IEEE Power Electronics Specialists Conference (PESC), 2, 1494 1498.

Pagano, D.J., Aracil, J., and Gordillo, F. (2005). Autonomous oscillation generation in the boost converter. in Proc. IFAC World Congress.

Ríos-Bolívar, M., Zinober, A., and Sira-Ramírez, H. (1996). Dynamical Sliding Mode Control via Adaptive Input-Output Linearization: A Backstepping Approach. Robust control via variable structure and Lyapunov techniques, 15.

Sanchís, P., Ursaea, A., Gubia, E., and Marroyo, L. (2005). Boost DC-AC Inverter: A New Control Strategy. IEEE Trans. Power Electronics, 20(2), 343-353. 
Sira-Ramírez, H. and Ríos-Bolívar, M. (1994). Sliding mode control of DCto-DC power converters via extended linearization. IEEE Trans. Circuits and Systems I: Fundamental Theory and Applications, 41(10), 652-661.

Sira-Ramírez, H., Ríos-Bolívar, M., and Zinober, A. (1997). Adaptive Dynamical Input-Output Linearization of DC to DC Power Converters: A Backstepping Approach. International Journal of Robust and Nonlinear Control, 7(3).

Tan, S., Lai, Y., and Tse, C. (2005). Adaptive control schemes for stabilizing switching frequency of sliding mode controlled power converters. in Proc. IEEE Power Electronics and Applications.

Vázquez, N., Cortés, D., Hernández, C., Álvarez, J., Arau, J., and Alvarez, J. (2003). A new nonlinear control strategy for the boost inverter. in Proc. IEEE Power Electronics Specialist Conference (PESC), 3, 1403-1407.

Zhu, L., Wang, K., Lee, F., Lai, J., and Ecostar, D. (2000). New start-up schemes for isolated full-bridge boost converters. In IEEE Applied Power Electronics Conference and Exposition, (APEC), volume 1. 